\title{
CONSTRUCTION OF FREE COMMUTATIVE INTEGRO-DIFFERENTIAL ALGEBRAS BY THE METHOD OF GRÖBNER-SHIRSHOV BASES
}

\author{
XING GAO, LI GUO, AND SHANGHUA ZHENG
}

\begin{abstract}
In this paper, we construct a canonical linear basis for free commutative integro-differential algebras by applying the method of Gröbner-Shirshov bases. We establish the CompositionDiamond Lemma for free commutative differential Rota-Baxter algebras of order $n$. We also obtain a weakly monomial order on these algebras, allowing us to obtain Gröbner-Shirshov bases for free commutative integro-differential algebras on a set. We finally generalize the concept of functional derivations to free differential algebras with arbitrary weight and generating sets from which to construct a canonical linear basis for free commutative integro-differential algebras.
\end{abstract}

\section{Contents}

1. Introduction 1

1.1. Integro-differential algebras 1

1.2. Gröbner-Shirshov bases 3

1.3. Outline of the paper 4

2. Free commutative integro-differential algebras 4

2.1. The definitions 4

2.2. Free differential Rota-Baxter algebras 5

2.3. Free commutative operated algebras 6

3. Weakly monomial order 9

4. Composition-Diamond lemma 14

5. Groöbner-Shirshov bases and free commutative integro-differential algebras 19

5.1. Gröbner-Shirshov basis 20

5.2. Bases for free commutative integro-differential algebras 23

$\begin{array}{ll}\text { References } & 27\end{array}$

\section{INTRODUCTION}

1.1. Integro-differential algebras. The algebraic study in analysis has a long history. The first monograph [30] of Ritt on algebraic study of differential equations appeared almost one hundred years ago. The concept of a differential algebra was abstracted from the Leibniz formula

$$
d(u v)=d(u) v+u d(v)
$$

in calculus. After the fundamental works of Ritt [31] and Kolchin [28], the theory of differential algebra has been expanded to a vast area of pure and applied mathematical study [13, 37]. The

Date: October 16, 2018.

2010 Mathematics Subject Classification. 16S15, 13P10, 16W99, 17A50, 12H05, 47G20 .

Key words and phrases. Differential algebra, Rota-Baxter algebra, integro-differential algebra, Gröbner-Shirshov basis, free algebra, shuffle product, mixable shuffle product. 
algebraic study of the integral analysis began with the concept of a Baxter algebra [3], later called a Rota-Baxter algebra. Here the basis of abstraction is the integration by parts formula,

$$
P(u) P(v)=P(u P(v))+P(P(u) v)+\lambda P(u v),
$$

rewritten in a form that only involves the integral operator $P$, defined by $P(u)(x):=\int_{a}^{x} u(t) d t$. The extra term parameterized by a constant $\lambda$ allows both the integral operator (when $\lambda=0$ ) and the summation operator (when $\lambda=1$ ), as well as quite a few other operators, to be encoded into one equation. Since then, Rota-Baxter algebra has found broad applications from combinatorics and number theory to classical Yang-Baxter equation and quantum field theory $[2,16,18,19,25$, 33, 34, 35].

Motivated by the close relationship between the differential and integral analysis as shown in the First Fundamental Theorem of Calculus, coordinated studied of differential algebra and Rota-Baxter algebra have emerged recently, beginning with the two simultaneously introduced concepts of a differential Rota-Baxter algebra and an integro-differential algebra.

The concept of a differential Rota-Baxter algebra [22] is a simple coupling of a differential operator $d$ of weight $\lambda$ :

$$
d(u v)=d(u) v+u d(v)+\lambda d(u) d(v), d(1)=0,
$$

with a Rota-Baxter operator $P$ of the same weight by the abstraction of the First Fundamental Theorem of Calculus

$$
d \circ P=\mathrm{id},
$$

where id is the identity map. On the other hand, the concept of an integro-differential algebra, first considered in the weight 0 case in [32] and in the general weight case in [23], also takes into account the intertwining relationship of the two operators in the original definition of the integration by parts formula

$$
P(d(u) P(v))=u P(v)-P(u v)-\lambda P(d(u) v) .
$$

We note that Eq. (5) implies Eq. (2) at the presence of Eq. (4) when $u$ is substituted by $P(u)$. Thus the variety of integro-differential algebras is the variety of differential Rota-Baxter algebras modulo extra conditions. See [23] for further details.

As in the case of studying any algebraic structures, the free objects play an important role in the study of previous algebras. While the construction of free differential algebras is straightforward in terms of differential monomials, the construction of free Rota-Baxter algebras is more involved. In fact, there are three constructions in the commutative case, with the first one given by Rota [33] through an internal construction, and an external one given by Cartier [12]. In [20], a construction is given by a generalization of the shuffle product, called the mixable shuffle product which is closely related to the quasi-shuffle product [27] in the study of multiple zeta values.

By composing the construction of free differential algebras followed by that of the free RotaBaxter algebras, free differential Rota-Baxter algebras were obtained in [22]. Because of the more intimate relationship of the differential and Rota-Baxter operators in an integro-differential algebra, it is more challenging to construct free objects in the corresponding category even by the previous remark on the variety of integro-differential algebras, free integro-differential algebras are quotients of free differential Rota-Baxter algebras modulo the relation given by Eq. (5). The first construction of free commutative integro-differential algebras was obtained in the recent paper [23]. There the construction makes essential use of an equivalent formulation of the condition in Eq. (5) for the integro-differential algebra. 
1.2. Gröbner-Shirshov bases. In this paper, we apply the method of Gröbner-Shirshov bases to give another construction of the free commutative integro-differential algebras on a set.

The method of Gröbner bases or Gröbner-Shirshov bases originated from the work of Buchburger [11] (for commutative polynomial algebras), Hironaka [26] (for infinite series algebras) and Shirshov [36] (for Lie algebras). It has since become a fundamental method in commutative algebra, algebraic geometry and computational algebra, and has been extended to many other algebraic structures, notably associative algebras $[4,5]$. In recent years, the method of GröbnerShirshov bases has been applied to a large number of algebraic structures to study problems on normal forms, word problems, rewriting systems, embedding theorems, extensions, growth functions and Hilbert series. See $[6,8,10]$ for further details.

This method also derives free objects in various categories, including the alternative constructions of free Rota-Baxter algebras and free differential Rota-Baxter algebras [7, 9]. The basic idea is to prove a composition-diamond lemma that achieves a rewriting procedure to reduce any element to certain "standard form". Then the set of elements in standard form is a basis of the free object.

We apply this method to construct a free commutative integro-differential algebra as the quotient of a free commutative differential Rota-Baxter algebra modulo the "hybrid" integral by part formula in Eq. (5). In order to do so, we would expect to first establish a Composition-Diamond Lemma for the free commutative differential Rota-Baxter algebra constructed in [22]. We should then prove that the ideal generated by the defining relation of integro-differential algebras in Eq. (5) has a Gröbner-Shirshov basis, thereby identifying a basis of a free commutative integrodifferential algebra as a canonical subset of the known basis of the free commutative differential Rota-Baxter algebra. All these depend on the choice of a suitable monomial order on the set of the basis elements of the free commutative differential Rota-Baxter algebra. However a moment's thought reveals that such a monomial order does not exist for this algebra. To overcome this difficulty, we consider this algebra as a filtered algebra with respect to the order of derivation and study the filtration pieces first. Even there, we have to get along with a weakly monomial order which fortunately suffices for our applications. So we are able to adapt the above process of Gröbner-Shirshov bases and obtain a canonical basis for each of the filtration pieces. We then check that this process is compatible with the filtration structure, allowing us to put these canonical bases for the filtration pieces together to form a canonical basis for the entire free commutative integro-differential algebra. The following is our main theorem

Theorem 1.1. (=Theorem 5.13) Let $X$ be a nonempty well-ordered set and $A:=\mathbf{k}\{X\}$. Let $\amalg(\mathbf{k}\{X\})=\amalg(\mathbf{k}[\Delta X])$, with the derivation $d$ and Rota-Baxter operator $P$, be the free commutative differential Rota-Baxter algebra of weight $\lambda$ on $X$. Let $I_{I D}$ be the differential Rota-Baxter ideal of $\amalg(\mathbf{k}\{X\})$ generated by

$$
S:=\{P(d(u) P(v))-u P(v)+P(u v)+\lambda P(d(u) v) \mid u, v \in \amalg(\mathbf{k}\{X\})\} .
$$

Let $A_{f}$ be the submodule of $A=\mathbf{k}\{X\}$ spanned by functional monomials. Then the composition

$$
\amalg(A)_{f}:=A \oplus\left(\bigoplus_{k \geq 0} A \otimes A_{f}^{\otimes k} \otimes A\right) \hookrightarrow \amalg(A) \rightarrow \amalg(A) / I_{I D}
$$

of the inclusion and the quotient map is a linear bijection. Thus $\amalg(A)_{f}$ gives an explicit construction of the free integro-differential algebra $\amalg(A) / I_{I D}$.

It is interesting to note that our approach of Gröbner-Shirshov bases gives a different construction of free commutative integro-differential algebras than those in [23]. While the construction 
in [23] has a transparent product formula, the construction here has a simple description as a submodule of the free differential Rota-Baxter algebra. By the uniqueness of the free objects, the two constructions yield isomorphic integro-differential algebras. Thus it would be interesting to compare the two constructions to reveal further the structure and properties of these free objects.

1.3. Outline of the paper. In Section 2, we first introduce the algebraic structures that lead up to $\lambda$-integro-differential algebras and then recall the construction of free objects for these algebraic structures, in particular the free commutative Rota-Baxter algebras and the free commutative differential Rota-Baxter algebras. In Section 3, we first give definitions related to differential Rota-Baxter monomials and then define a weakly monomial order on differential Rota-Baxter monomials of order $n$. In Section 4, we start with defining various kinds of compositions and then establish the Composition-Diamond Lemma for the $n$-th order free commutative differential Rota-Baxter algebra. In Section 5, we consider a finite set $X$ and obtain a Gröbner-Shirshov basis for the defining ideal of a free commutative order $n$ integro-differential algebra on $X$ and thus obtain an explicitly defined basis for this free object. Then as mentioned above, we put the order $n$ pieces together as a direct system to obtain a basis for the free commutative integro-differential algebra on $X$. We then use a finiteness argument to treat the case when $X$ is any well-ordered set.

\section{Free commutative integro-DifFerential algebras}

We recall the definitions of algebras with various differential and integral operators and the constructions of the free objects in the corresponding categories.

2.1. The definitions. We recall the algebraic structures considered in this paper. We also introduce variations with bounded derivation order that will be needed later.

Definition 2.1. Let $\mathbf{k}$ be a unitary commutative ring. Let $\lambda \in \mathbf{k}$ be fixed.

(a) A differential k-algebra of weight $\lambda$ (also called a $\lambda$-differential k-algebra) is a unitary associative k-algebra $R$ together with a linear operator $d: R \rightarrow R$ such that

$$
d(1)=0, d(u v)=d(u) v+u d(v)+\lambda d(u) d(v) \text { for all } u, v \in R .
$$

Such an algebra $(R, d)$ is said of order $n$, where $n \geq 1$, if $d^{n}=0$.

(b) A Rota-Baxter k-algebra of weight $\lambda$ is an associative $\mathbf{k}$-algebra $R$ together with a linear operator $P: R \rightarrow R$ such that

$$
P(u) P(v)=P(u P(v))+P(P(u) v)+\lambda P(u v) \text { for all } u, v \in R .
$$

(c) A differential Rota-Baxter k-algebra of weight $\lambda$ (also called a $\lambda$-differential RotaBaxter k-algebra) is a differential k-algebra $(R, d)$ of weight $\lambda$ and a Rota-Baxter operator $P$ of weight $\lambda$ such that

$$
d \circ P=\text { id }
$$

(d) An integro-differential k-algebra of weight $\lambda$ (also called a $\lambda$-integro-differential kalgebra) is a differential $\mathbf{k}$-algebra $(R, d)$ of weight $\lambda$ with a linear operator $P: R \rightarrow R$ that satisfies Eq. (8) and such that

$$
P(d(u) P(v))=u P(v)-P(u v)-\lambda P(d(u) v) \text { for all } u, v \in R .
$$


2.2. Free differential Rota-Baxter algebras. We first recall the construction of free commutative differential algebras and introduce their order $n$ variations.

Theorem 2.2. Let $X$ be a set.

(a) Let $\Delta X=\left\{x^{(n)} \mid x \in X, n \geq 0\right\}$ and let $\mathbf{k}\{X\}=\mathbf{k}[\Delta X]$ be the free commutative algebra on the set $\Delta X$. Define $d_{X}: \mathbf{k}[\Delta X] \rightarrow \mathbf{k}[\Delta X]$ as follows. Let $w=u_{1} \cdots u_{k}, u_{i} \in \Delta X, 1 \leq i \leq k$, be a commutative word from the alphabet set $\Delta X$. If $k=1$, so that $w=x^{(n)} \in \Delta X$, define $d_{X}(w)=x^{(n+1)}$. If $k>1$, recursively define

$$
d_{X}(w)=d_{X}\left(u_{1}\right) u_{2} \cdots u_{k}+u_{1} d_{X}\left(u_{2} \cdots u_{k}\right)+\lambda d_{X}\left(u_{1}\right) d_{X}\left(u_{2} \cdots u_{k}\right) .
$$

Further define $d_{X}(1)=0$ and then extend $d_{X}$ to $\mathbf{k}[\Delta(X)]$ by linearity. Then $\left(\mathbf{k}[\Delta X], d_{X}\right)$ is the free commutative differential algebra of weight $\lambda$ on the set $X$.

(b) For a given $n \geq 1$, let $\Delta X^{(n+1)}:=\left\{x^{(k)} \mid x \in X, k \geq n+1\right\}$. Then $\mathbf{k}\{X\} \Delta X^{(n+1)}$ is the differential ideal $I_{n}$ of $\mathbf{k}\{X\}$ generated by the set $\left\{x^{(n+1)} \mid x \in X\right\}$. The quotient $\mathbf{k}\{X\} / I_{n}$ has a canonical basis given by $\Delta_{n} X:=\left\{x^{(k)} \mid k \leq n\right\}$.

Proof. Item (a) is from [22] and Item (b) is a direct consequence.

For a set $Y$, let $C(Y)$ denote the free commutative monoid on $Y$. Thus elements in $C(Y)$ are commutative words, plus the identity 1 , from the alphabet set $Y$. Then $C(\Delta X)\left(\operatorname{resp} . C\left(\Delta_{n} X\right)\right)$ is a linear basis of $\mathbf{k}[\Delta X]$ (resp. $\mathbf{k}\left[\Delta_{n} X\right]$ ).

We next recall the construction of free commutative Rota-Baxter algebras in terms of mixable shuffles $[20,21]$. The mixable shuffle product is shown to be the same as the quasi-shuffle product of Hoffman [15, 25, 27]. Let $A$ be a commutative k-algebra. Define

$$
\amalg(A)=\bigoplus_{k \geq 0} A^{\otimes(k+1)}=A \oplus A^{\otimes 2} \oplus \cdots .
$$

Let $\mathfrak{a}=a_{0} \otimes \cdots \otimes a_{m} \in A^{\otimes(m+1)}$ and $\mathfrak{b}=b_{0} \otimes \cdots \otimes b_{n} \in A^{\otimes(n+1)}$. If $m=0$ or $n=0$, define

$$
\mathfrak{a} \diamond \mathfrak{b}= \begin{cases}\left(a_{0} b_{0}\right) \otimes b_{1} \otimes \cdots \otimes b_{n}, & m=0, n>0, \\ \left(a_{0} b_{0}\right) \otimes a_{1} \otimes \cdots \otimes a_{m}, & m>0, n=0, \\ a_{0} b_{0}, & m=n=0 .\end{cases}
$$

If $m>0$ and $n>0$, inductively (on $m+n$ ) define

$$
\begin{aligned}
\mathfrak{a} \diamond \mathfrak{b}=\left(a_{0} b_{0}\right) \otimes & \left(a_{1} \otimes a_{2} \otimes \cdots \otimes a_{m}\right) \diamond\left(1 \otimes b_{1} \otimes \cdots \otimes b_{n}\right) \\
& +\left(1 \otimes a_{1} \otimes \cdots \otimes a_{m}\right) \diamond\left(b_{1} \otimes \cdots \otimes b_{n}\right) \\
& \left.+\lambda\left(a_{1} \otimes \cdots \otimes a_{m}\right) \diamond\left(b_{1} \otimes \cdots \otimes b_{n}\right)\right) .
\end{aligned}
$$

Extending by additivity, we obtain a k-bilinear map

$$
\diamond: \amalg(A) \times \amalg(A) \rightarrow \amalg(A) .
$$

Alternatively,

$$
\mathfrak{a} \diamond \mathfrak{b}=\left(a_{0} b_{0}\right) \otimes\left(\overline{\mathfrak{a}}_{ш_{\lambda}} \overline{\mathfrak{b}}\right),
$$

where $\overline{\mathfrak{a}}=a_{1} \otimes \cdots \otimes a_{m}, \overline{\mathfrak{b}}=b_{1} \otimes \cdots \otimes b_{n}$ and $\omega_{\lambda}$ is the mixable shuffle (quasi-shuffle) product of weight $\lambda[19,20,27]$, which specializes to the shuffle product $ш$ when $\lambda=0$.

Define a k-linear endomorphism $P_{A}$ on $\amalg(A)$ by assigning

$$
P_{A}\left(x_{0} \otimes x_{1} \otimes \cdots \otimes x_{n}\right)=\mathbf{1}_{A} \otimes x_{0} \otimes x_{1} \otimes \cdots \otimes x_{n},
$$


for all $x_{0} \otimes x_{1} \otimes \cdots \otimes x_{n} \in A^{\otimes(n+1)}$ and extending by additivity. Let $j_{A}: A \rightarrow \amalg(A)$ be the canonical inclusion map.

Theorem 2.3. ([20, 21])

(a) The pair $\left(\amalg(A), P_{A}\right)$, together with the natural embedding $j_{A}: A \rightarrow \amalg(A)$, is the free commutative Rota-Baxter $\mathbf{k}$-algebra on A of weight $\lambda$. In other words, for any Rota-Baxter $\mathbf{k}$-algebra $(R, P)$ and any k-algebra map $\varphi: A \rightarrow R$, there exists a unique Rota-Baxter k-algebra homomorphism $\tilde{\varphi}:\left(\amalg(A), P_{A}\right) \rightarrow(R, P)$ such that $\varphi=\tilde{\varphi} \circ j_{A}$ as $\mathbf{k}$-algebra homomorphisms.

(b) Let $Y$ be a set and let $\mathbf{k}[Y]$ be the free commutative algebra on $Y$. The pair $\left(\amalg(Y), P_{Y}\right):=$ $\left(\amalg(\mathbf{k}[Y]), P_{\mathbf{k}[Y]}\right)$, together with the natural embedding $j_{Y}: Y \rightarrow \mathbf{k}[Y] \rightarrow \amalg(\mathbf{k}[Y])$, is the free commutative Rota-Baxter $\mathbf{k}$-algebra of weight $\lambda$ on $Y$.

Since $\diamond$ is compatible with the multiplication in $A$, we will often suppress the symbol $\diamond$ and simply denote $x y$ for $x \diamond y$ in $\amalg(A)$, unless there is a danger of confusion.

A linear basis of $\amalg(\mathbf{k}[Y])$ is given by

$$
\mathfrak{B}(Y):=\left\{x_{0} \otimes \cdots \otimes x_{k} \mid x_{i} \in C(Y), 1 \leq i \leq k, k \geq 0\right\},
$$

called the set of Rota-Baxter monomials in $Y$. The integer $\operatorname{dep}\left(x_{0} \otimes \cdots \otimes x_{k}\right):=k+1$ is called the depth of $x_{0} \otimes \cdots \otimes x_{k}$. To simplify notations, we also let $P$ denote $P_{\mathbf{k}[Y]}$. Then $1 \otimes u$ and $P(u)$ stand for the same element and will be be used as convenience in this paper.

We now put the differential and Rota-Baxter algebra structures together. Let $\left(A, d_{0}\right)$ be a commutative differential k-algebra of weight $\lambda$. Extend $d_{0}$ to $\amalg(A)$ by

$$
\begin{aligned}
& d_{A}\left(x_{0} \otimes x_{1} \otimes \ldots \otimes x_{k}\right) \\
& \quad=\quad d_{0}\left(x_{0}\right) \otimes x_{1} \otimes \ldots \otimes x_{k}+x_{0} x_{1} \otimes x_{2} \otimes \ldots \otimes x_{k}+\lambda d_{0}\left(x_{0}\right) x_{1} \otimes x_{2} \otimes \ldots \otimes x_{k}, \quad k \geq 0 .
\end{aligned}
$$

Theorem 2.4. ([22]) Let $X$ be a set and let $\mathbf{k}[\Delta X]$ be the free commutative differential algebra of weight $\lambda$ on $X$ in Theorem 2.2.(a). The triple ( $\left.\mathrm{W}(\mathbf{k}[\Delta X]), d_{\mathbf{k}[\Delta X]}, P_{\mathbf{k}[\Delta X]}\right)$, together with $j_{X}: X \rightarrow$ $\Delta X \rightarrow \amalg(\mathbf{k}[\Delta X])$, is the free commutative differential Rota-Baxter $\mathbf{k}$-algebra of weight $\lambda$ on $X$.

Apply the notations in Eq. (13) to $Y:=\Delta X$. The set

$$
\mathfrak{B}(\Delta X):=\left\{u_{0} \otimes \cdots \otimes u_{k} \mid u_{i} \in C(\Delta X), 0 \leq i \leq k, k \geq 0\right\}
$$

is a k-basis of the free commutative differential Rota-Baxter algebra $\amalg(\Delta X)$, called the set of differential Rota-Baxter (DRB) monomials on $X$.

Similarly with $Y:=\Delta_{n} X, n \geq 1, \mathcal{B}\left(\Delta_{n} X\right)$ is a basis of $\amalg\left(\Delta_{n} X\right)$ and is called the set of DRB monomials of order $n$ on $X$. We note that in $\amalg\left(\mathbf{k}\left[\Delta_{n} X\right]\right)$, the property $d^{n+1}(u)=0$ only applies to $u \in X$, but not to tensors of length greater than two. For example, taking $n=1$, then $d^{2}(x)=0$, but $d(1 \otimes x)=x$ and hence $d^{2}(1 \otimes x)=d(x)=x^{(1)} \neq 0$.

2.3. Free commutative operated algebras. We now construct the free commutative operated algebra on a set $X$ that has the free commutative (differential) Rota-Baxter algebra as a quotient. At the same time, the explicit construction $\amalg(X)$ of free commutative Rota-Baxter algebra in Theorem 2.3 can be realized on a submodule of the free commutative operated algebra spanned by reduced words under a rewriting rule defined by the Rota-Baxter axiom.

This construction is parallel to that of the free (noncommutative) operated algebra on a set in $[9,17,19,24]$. See [29] for the non-unitary case. 
Definition 2.5. A commutative operated monoid with operator set $\Omega$ is a commutative monoid $G$ together with maps $\alpha_{\omega}: G \rightarrow G, \omega \in \Omega$. A homomorphism between commutative operated monoids $\left(G,\left\{\alpha_{\omega}\right\}_{\omega}\right)$ and $\left(H,\left\{\beta_{\omega}\right\}_{\omega}\right)$ is a monoid homomorphism $f: G \rightarrow H$ such that $f \circ \alpha_{\omega}=$ $\beta_{\omega} \circ f$ for $\omega \in \Omega$.

We next construct the free objects in the category of commutative operated monoids.

Fix a set $Y$. We define monoids $\mathfrak{C}_{n}:=\mathfrak{C}_{n}(Y)$ for $n \geq 0$ by a recursion. First denote $\mathfrak{C}_{0}:=C(Y)$. Let $\lfloor C(Y)\rfloor_{\omega}:=\left\{\lfloor u\rfloor_{\omega} \mid u \in C(Y)\right\}, \omega \in \Omega$, be disjoint sets in bijection with and disjoint from $C(Y)$. Then define

$$
\mathfrak{c}_{1}:=C\left(Y \sqcup\left(\sqcup_{\omega \in \Omega}\lfloor C(Y)\rfloor_{\omega}\right)\right) .
$$

Note that elements in $\lfloor C(Y)\rfloor_{\omega}$ are only symbols indexed by elements in $C(Y)$. For example, $\lfloor 1\rfloor_{\omega}$ is not the identity, but a new symbol. The inclusion $Y \hookrightarrow Y \sqcup\left(\sqcup_{\omega \in \Omega}\left\lfloor\mathfrak{C}_{0}\right\rfloor_{\omega}\right)$ induces a monomorphism $i_{0,1}: \mathfrak{C}_{0}=C(Y) \hookrightarrow \mathfrak{C}_{1}=C\left(Y \sqcup\left(\sqcup_{\omega}\left\lfloor\mathfrak{C}_{0}\right\rfloor_{\omega}\right)\right)$ of free commutative monoids through which we identify $\mathfrak{C}_{0}$ with its image in $\mathfrak{C}_{1}$. Inductively assume that $\mathfrak{C}_{n-1}$ have been defined for $n \geq 2$ and that the embedding

$$
i_{n-2, n-1}: \mathfrak{C}_{n-2} \rightarrow \mathfrak{C}_{n-1}
$$

has been obtained. We then define

$$
\mathfrak{C}_{n}:=C\left(Y \sqcup\left(\sqcup_{\omega}\left\lfloor\mathfrak{C}_{n-1}\right\rfloor_{\omega}\right)\right) .
$$

We also have the injection

$$
\left\lfloor\mathfrak{C}_{n-2}\right\rfloor_{\omega} \hookrightarrow\left\lfloor\mathfrak{C}_{n-1}\right\rfloor_{\omega}, \omega \in \Omega .
$$

Thus by the freeness of $\widetilde{C}_{n-1}=C\left(Y \sqcup\left(\sqcup_{\omega}\left\lfloor\mathfrak{C}_{n-2}\right\rfloor_{\omega}\right)\right)$ as a free commutative monoid, we have

$$
\mathfrak{C}_{n-1}=C\left(Y \sqcup\left(\sqcup_{\omega}\left\lfloor\mathfrak{C}_{n-2}\right\rfloor_{\omega}\right)\right) \hookrightarrow C\left(Y \sqcup\left(\sqcup_{\omega}\left\lfloor\mathfrak{C}_{n-1}\right\rfloor_{\omega}\right)\right)=\mathfrak{C}_{n} .
$$

We finally define the commutative monoid

$$
\mathfrak{C}(Y):=\bigcup_{n \geq 0} \mathfrak{c}_{n}=\underset{\lim }{\longrightarrow} \mathfrak{c}_{n} .
$$

Elements in $\mathfrak{C}(Y)$ are called bracketed monomials in $Y$. Defining

$$
\lfloor\rfloor_{\omega}: \mathfrak{C}(Y) \rightarrow \mathfrak{C}(Y), u \mapsto\lfloor u\rfloor_{\omega}, \omega \in \Omega,
$$

$\left.\left(\mathfrak{C}(Y),\{\mathrm{L}\rfloor_{\omega}\right\}_{\omega}\right)$ is a commutative operated monoid and its linear span $\left.(\mathbf{k} \mathfrak{C}(Y), \mathrm{L}\rfloor_{\omega}\right)$ is a commutative (unitary) operated k-algebra.

Proposition 2.6. Let $j_{Y}: Y \rightarrow \mathfrak{C}(Y)$ be the natural embedding.

(a) The triple $\left.\left(\mathfrak{C}(Y),\{\mathrm{L}\rfloor_{\omega}\right\}_{\omega}, j_{Y}\right)$ is the free commutative operated monoid on $Y$. More precisely, for any commutative operated monoid $G$ and set map $f: Y \rightarrow G$, there is a unique extension of $f$ to a homomorphism $\bar{f}: \mathfrak{C}(Y) \rightarrow G$ of operated monoids.

(b) The triple $\left.\left(\mathbf{k} \mathfrak{C}(Y),\{\mathrm{L}\rfloor_{\omega}\right\}_{\omega}, j_{Y}\right)$ is the free commutative operated unitary $\mathbf{k}$-algebra on $Y$. More precisely, for any commutative k-algebra $R$ and set map $f: Y \rightarrow R$, there is a unique extension of $f$ to a homomorphism $\bar{f}: \mathbf{k} \mathfrak{C}(Y) \rightarrow R$ of operated $\mathbf{k}$-algebras.

Proof. We only need to show that $\mathfrak{C}(Y)$ is a free commutative operated monoid. The proof is similar to the noncommutative case $[17,19]$, so we just give a sketch.

Let a commutative operated monoid $\left(G,\left\{\alpha_{\omega}\right\}_{\omega}\right)$ and a map $f: Y \rightarrow G$ be given. Then by the universal property of $\mathfrak{C}_{0}:=C(Y)$, there is a unique monoid homomorphism $f_{0}: \mathfrak{C}_{0} \rightarrow G$ extending $f$. Then $f_{0}$ extends uniquely to

$$
f_{1}:\left\lfloor\mathfrak{C}_{0}\right\rfloor_{\omega} \rightarrow G, \quad\lfloor u\rfloor_{\omega} \mapsto \alpha_{\omega}\left(f_{0}(u)\right), u \in \mathfrak{C}_{0},
$$


such that $\left(f_{1} \circ\lfloor\rfloor_{\omega}\right)(u)=\left(\alpha_{\omega} \circ f_{1}\right)(u), \omega \in \Omega$, when defined. We then further get a monoid homomorphism

$$
f_{1}: \mathfrak{C}_{1}:=C\left(Y \sqcup\left(\sqcup_{\omega}\left\lfloor\mathfrak{C}_{0}\right\rfloor_{\omega}\right)\right) \rightarrow G
$$

By induction on $n \geq 0$ we obtain a unique $f_{n}: \mathfrak{C}_{n} \rightarrow G, n \geq 0$, compatible with the direct system, yielding the unique homomorphism $\bar{f}: \mathfrak{C}(Y) \rightarrow G$ of operated monoids.

By the universal property of $\mathbf{k} \mathfrak{C}(Y)$, we obtain the following conclusion from general principles of universal algebra [1,14].

Proposition 2.7. Let $\Omega=\{d, P\}$ and denote $d(u):=\lfloor u\rfloor_{d}, P(u):=\lfloor u\rfloor_{P}$. Let $I_{D R B}$ be the operated ideal of $\mathbf{k} \mathfrak{C}(Y)$ generated by the set

$$
\left\{\begin{array}{l}
d(u v)-d(u) v-u d(v)-\lambda d(u) d(v), \\
P(u) P(v)-P(u P(v))-P(P(u) v)-\lambda P(u v), \mid u, v \in \mathbb{C}(Y) \\
(d \circ P)(u)=u
\end{array}\right\} .
$$

Then the quotient operated algebra $\mathbf{k} \mathfrak{C}(Y) / I_{D R B}$, with the quotient of the operator $d$ and $P$, is the free commutative differential Rota-Baxter algebra.

Combining Proposition 2.7 with Theorem 2.3, we have

Proposition 2.8. The natural embedding

$$
\amalg(\mathbf{k}[\Delta X]) \rightarrow \mathbf{k} \mathfrak{C}(\Delta X), \quad x_{0} \otimes x_{1} \otimes \cdots \otimes x_{k} \mapsto x_{0} P\left(x_{1} P\left(\cdots P\left(x_{k}\right) \cdots\right)\right)
$$

composed with the quotient map $\rho: \mathbf{k} \mathfrak{C}(\Delta X) \rightarrow \mathbf{k} \mathfrak{C}(\Delta X) / I_{D R B}$ gives a linear bijection (in fact, an isomorphism of differential Rota-Baxter algebras)

$$
\theta: \amalg(\mathbf{k}[\Delta X]) \rightarrow \mathbf{k} \mathfrak{C}(\Delta X) / I_{D R B} .
$$

Through $\theta$, we can identify the basis $\mathfrak{B}(\Delta X)$ of $\amalg(\mathbf{k} \Delta X)$ with its image in $\mathbf{k} \mathfrak{C}(\Delta X)$ :

$$
u_{0} \otimes u_{1} \otimes \cdots \otimes u_{k} \leftrightarrow u_{0}\left\lfloor u_{1}\left\lfloor\cdots\left\lfloor u_{k}\right\rfloor \cdots\right\rfloor\right\rfloor \leftrightarrow u_{0} P\left(u_{1} P\left(\cdots P\left(u_{k}\right) \cdots\right)\right) .
$$

Thus we also use $P$ for $P_{\Delta X}$ on $\amalg(\mathbf{k}[\Delta X])$ and $d^{\ell}(x)=x^{(\ell)}$ for $x \in X$ and $\ell \geq 0$.

As a consequence of Proposition 2.8, we have

Corollary 2.9. Let $n \geq 1$. Let $I_{D R B, n}$ be the operated ideal of $\mathfrak{C}(X)$ generated by $I_{D R B}$ together with the set $\left\{x^{(n+1)}=d^{n+1}(x) \mid x \in X\right\}$. The natural embedding

$$
\amalg\left(\mathbf{k}\left[\Delta_{n} X\right]\right) \rightarrow \mathbf{k} \mathbb{C}(X), \quad x_{0} \otimes x_{1} \otimes \cdots \otimes x_{k} \mapsto x_{0} P\left(x_{1} P\left(\cdots P\left(x_{k}\right) \cdots\right)\right)
$$

composed with the quotient map $\rho: \mathbf{k} \mathfrak{C}(Y) \rightarrow \mathbf{k} \mathfrak{C}(Y) / I_{D R B, n}$ gives a linear bijection

$$
\theta_{n}: \amalg\left(\mathbf{k}\left[\Delta_{n} X\right]\right) \rightarrow \mathbf{k} \mathbb{C}(X) / I_{D R B, n} .
$$

Proof. The map $\theta_{n}$ is obtained by starting from the isomorphism $\theta: \amalg(\mathbf{k}[\Delta X]) \cong \mathbf{k} \subseteq(X) / I_{D R B}$ and then taking the quotients of both the domain and range by the operated ideal generated by $d^{n+1}(x), x \in X$. Since $\theta$ restricted to the identity on $X$. The corollary follows.

Define the reduction map

$$
\operatorname{Red}:=\operatorname{Red}_{n}:=\theta_{n}^{-1} \circ \rho: \mathbf{k} \mathfrak{C}(X) \rightarrow \mathbf{k} \mathbb{C}(Y) / I_{D R B, n} \rightarrow \amalg\left(\mathbf{k}\left[\Delta_{n} X\right]\right) .
$$

It reduces any bracketed monomial to a DRB monomial. For example, if $u, v \in C(X)$, then

$$
\operatorname{Red}(\lfloor u\rfloor v\rfloor)=1 \otimes u \otimes v+1 \otimes v \otimes u+\lambda \otimes u v .
$$




\section{WEAKLY MONOMIAL ORDER}

In this section, we will give a weak form of the monomial order on filtered pieces of the set of differential Rota-Baxter monomials. It will be sufficient for us to establish the compositiondiamond lemma for integro-differential algebras.

Let $Y$ be a set with well order $\leq_{Y}$. Define the length-lexicographic order $\leq_{Y, \text { lex }}^{*}$ on the free monoid $M(Y)$ by

$$
u<_{Y, \text { lex }}^{*} v \Leftrightarrow\left\{\begin{array}{l}
\ell<m, \\
\text { or } \ell=m \text { and } \exists 1 \leq i_{0} \leq \ell \text { such that } u_{i}=v_{i} \text { for } 1 \leq i<i_{0} \text { and } u_{i_{0}}<v_{i_{0}},
\end{array}\right.
$$

where $u=u_{1} \cdots u_{\ell}$ and $v=v_{1} \cdots v_{m}$ with $u_{i} \in Y, 1 \leq i \leq \ell, v_{j} \in Y, 1 \leq j \leq m, m, n \geq 1$. It is well-known [1] that $\leq_{Y, \text { lex }}^{*}$ is still a well order. An element $1 \neq u$ of the free commutative monoid $C(Y)$ can be uniquely expressed as

$$
u=u_{0}^{j_{0}} \cdots u_{k}^{j_{k}} \text {, where } u_{0}, \cdots, u_{k} \in Y, j_{0}, \cdots, j_{k} \in \mathbb{Z}_{\geq 1} \text { and } u_{0}>\cdots>u_{k} .
$$

This expression is called the standard form of $u$. If $k=-1$, we take $u \in \mathbf{k}$ by convention.

Any $1 \neq u \in C(Y)$ can also be expressed uniquely as

$$
u=u_{1} \cdots u_{\ell}, u_{1} \geq u_{2} \geq \cdots \geq u_{\ell} \in Y .
$$

With this notation, $C(Y)$ can be identified with a subset of the free monoid $M(Y)$ on $Y$. Then the well order $<_{Y, \text { lex }}^{*}$ on $M(Y)$ restricts to a well order on $C(Y)$.

Lemma 3.1. Let $\left(Y, \leq_{Y}\right)$ is a well-ordered set and $u, v \in C(Y)$. If $u<v$, then $u w \leq_{Y, \text { lex }}^{*} v w$ for $w \in C(Y)$.

Proof. Such a result is well-known for free noncommutative monoid. The proof for the commutative case is different and we sketch a proof for completeness.

From the standard decomposition of $u \in C(Y)$ in Eq. (20), $u$ can be expressed uniquely as a function

$$
f:=f_{u}: Y \rightarrow \mathbb{Z}_{\geq 0}, f_{u}(y)= \begin{cases}j_{i}, & y=u_{i}, 1 \leq i \leq k, \\ 0, & \text { otherwise. }\end{cases}
$$

Thus $C(Y)$ can be identified with

$$
\mathcal{F}:=\left\{f: Y \rightarrow \mathbb{Z}_{\geq 0} \mid \operatorname{Supp}(f):=Y \backslash f^{-1}(0) \text { is finite }\right\}
$$

with $1 \in C(Y)$ corresponding to $f_{1} \equiv 0$. Denote $\operatorname{deg}(f):=\sum_{y \in Y} f(y)$. Under this identification, the order $\leq_{Y, \text { lex }}^{*}$ on $C(Y)$ is identified with the order $\leq$ on $\mathcal{F}$ defined by

$f<g \Leftrightarrow\left\{\begin{array}{l}\operatorname{deg}(f)<\operatorname{deg}(g) \\ \operatorname{or} \operatorname{deg}(f)=\operatorname{deg}(g) \text { and } \exists y_{0} \in Y \text { such that } f(y)=g(y) \text { for } y<y_{0} \text { and } f\left(y_{0}\right)<g\left(y_{0}\right) .\end{array}\right.$

Let $u, v, w \in C(Y)$ be given. We apply the identification of $u, v, w$ with $f_{u}, f_{v}, f_{w} \in \mathcal{F}$ given in Eq (21). We note that $f_{u w}=f_{u}+f_{w}$ and $f_{v w}=f_{v}+f_{w}$. Thus we have

$\operatorname{deg}\left(f_{u w}\right)=\operatorname{deg}\left(f_{u}\right)+\operatorname{deg}\left(f_{w}\right), \operatorname{deg}\left(f_{v w}\right)=\operatorname{deg}\left(f_{v}\right)+\operatorname{deg}\left(f_{w}\right)$, and $f_{u}(y)<f_{v}(y) \Leftrightarrow f_{u w}(y)<f_{v w}(y)$.

Then it follows that $f_{u}<f_{v}$ if and only if $f_{u w}<f_{v w}$. This proves the lemma.

For a set $X$, recall that $\Delta X=\left\{x^{(k)} \mid x \in X, k \geq 0\right\}$ and $\Delta_{n} X:=\left\{x^{(k)} \mid x \in X, 0 \leq k \leq n\right\}$ for $n \geq 0$. Then $C\left(\Delta_{n} X\right), n \geq 0$, define an increasing filtration on $C(\Delta X)$ and hence give a filtration $\mathcal{B}\left(\Delta_{n} X\right) \subseteq \mathcal{B}(\Delta X)$. Elements of $\mathcal{B}\left(\Delta_{n} X\right)$ are called DRB monomials of order $n$. 
Definition 3.2. Let $X$ be a set, $\star$ a symbol not in $X$ and $\Delta_{n} X^{\star}:=\Delta_{n}(X \cup\{\star\})$.

(a) By a $\star$-DRB monomial on $\Delta_{n} X$, we mean any expression in $\mathcal{B}\left(\Delta_{n} X^{\star}\right)$ with exactly one occurrence of $\star$. The set of all $\star$-DRB monomials on $\Delta_{n} X$ is denoted by $\mathcal{B}^{\star}\left(\Delta_{n} X\right)$.

(b) For $q \in \mathcal{B}^{\star}\left(\Delta_{n} X\right)$ and $u \in \mathcal{B}\left(\Delta_{n} X\right)$, we define

$$
\left.q\right|_{u}:=\left.q\right|_{\star \mapsto u}
$$

to be the bracketed monomial in $\mathfrak{C}\left(\Delta_{n} X\right)$ obtained by replacing the letter $\star$ in $q$ by $u$, and call $\left.q\right|_{u}$ a $u$-monomial on $\Delta_{n} X$.

(c) Further, for $s=\sum_{i} c_{i} u_{i} \in \mathbf{k} \mathcal{B}\left(\Delta_{n} X\right)$, where $c_{i} \in \mathbf{k}, u_{i} \in \mathcal{B}\left(\Delta_{n} X\right)$ and $q \in \mathcal{B}^{\star}\left(\Delta_{n} X\right)$, we define

$$
\left.q\right|_{s}:=\left.\sum_{i} c_{i} q\right|_{u_{i}}
$$

which is in $\mathbf{k} \mathfrak{C}\left(\Delta_{n} X\right)$.

We note that a $\star$-DRB monomial $q$ is a DRB monomial in $\Delta_{n} X^{\star}$ while its substitution $\left.q\right|_{u}$ might not be a DRB monomials. For example, for $q=P\left(x_{1}\right) \star \in \mathcal{B}\left(\Delta_{n} X^{\star}\right)$ and $u=P\left(x_{2}\right) \in \mathcal{B}\left(\Delta_{n} X\right)$ where $x_{1}, x_{2} \in X$, the $u$-monomial $\left.q\right|_{u}=P\left(x_{1}\right) P\left(x_{2}\right)$ is no longer in $\mathcal{B}\left(\Delta_{n} X\right)$.

Lemma 3.3. Let $S$ be a subset of $\mathbf{k} \mathfrak{C}\left(\Delta_{n}(X)\right)$ and $\operatorname{Id}(\mathrm{S})$ be the operated ideal of $\mathbf{k} \mathfrak{C}\left(\Delta_{n}(X)\right)$ generated by $S$. Then

$$
\operatorname{Id}(\mathrm{S})=\left\{\left.\sum_{i=1}^{k} c_{i} q_{i}\right|_{s_{i}} \mid c_{i} \in \mathbf{k}, q_{i} \in \mathfrak{C}^{\star}\left(\Delta_{n} X\right), s_{i} \in S, 1 \leq i \leq k, k \geq 1\right\} .
$$

Proof. It is easy to see that the right hand side is contained in the left side. On the other hand, the right hand side is already an operated ideal of $\mathbf{k} \mathfrak{C}\left(\Delta_{n}(X)\right)$ containing $S$.

Definition 3.4. If $q=\left.p\right|_{d^{\ell}(\star)}$ for some $p \in \mathcal{B}^{\star}\left(\Delta_{n} X\right)$ and $\ell \in \mathbb{Z}_{\geq 1}$, then we call $q$ a type $\mathbf{I} \star-D R B$ monomial. Let $\mathcal{B}_{I}^{\star}\left(\Delta_{n} X\right)$ denote the set of type $\mathrm{I} \star$-DRB monomials on $\Delta_{n} X$ and call

$$
\mathcal{B}_{I I}^{\star}\left(\Delta_{n} X\right):=\mathcal{B}^{\star}\left(\Delta_{n} X\right) \backslash \mathcal{B}_{I}^{\star}\left(\Delta_{n} X\right)
$$

the set of type II $\star$-DRB monomials.

Lemma 3.5. Any element $q \in \mathcal{B}^{\star}\left(\Delta_{n} X\right)$ is one of the following three forms

(a) $q \in \mathcal{B}_{I}^{\star}\left(\Delta_{n} X\right)$, or

(b) $q=s \star t$ with $s \in C\left(\Delta_{n} X\right)$ and $t \in \mathcal{B}\left(\Delta_{n} X\right)$, or

(c) $q=s P(p)$ for some $s \in C\left(\Delta_{n} X\right)$ and $p \in \mathcal{B}_{I I}^{\star}\left(\Delta_{n} X\right)$.

Proof. Any element $q \in \mathcal{B}^{\star}\left(\Delta_{n} X\right)$ is of the form $u_{0} \otimes u_{1} \otimes \cdots \otimes u_{k}$ with $u_{i} \in C\left(\Delta_{n} X\right), 1 \leq i \leq k$, except a unique $u_{i}$ which is in $C\left(\Delta_{n} X^{\star}\right)$ with exactly one occurrence of $\star$. In turn, this unique $u_{i} \in C\left(\Delta_{n} X^{\star}\right)$ is of the form $u_{i 1} \cdots u_{i m}$ with $u_{i j} \in \Delta_{n} X, 1 \leq j \leq m$, except a unique $u_{i j}$ which is in $\Delta_{n} X^{\star}$ with exactly one occurrence of $\star$. Thus this unique $u_{i j} \in \Delta_{n} X^{\star}$ is of the for $d^{\ell}(\star)$ for some $\ell \geq 0$. If $\ell \geq 1$, then $q$ is of type I. If $\ell=0$, then $d^{\ell}(\star)=\star$. So if $i=0$, namely this $\star$ is in $u_{0}$, then $q=\left(u_{01} \cdots u_{0(j-1)} \star u_{0(j+1)} \cdots u_{0 m}\right) \otimes u_{1} \otimes \cdots u_{k}$ is of the form $s \star t$ with $s=u_{01} \cdots u_{0(j-1)} u_{0(j+1)} \cdots u_{0 m}$ and $t=1 \otimes u_{2} \cdots u_{k}$. If $i \geq 1$, then $q=s P(p)$, where $p:=u_{2} \otimes \cdots u_{k} \in \mathcal{B}_{I I}^{\star}\left(\Delta_{n} X\right)$. This proves the lemma.

Definition 3.6. Let $X$ be a set, $\star_{1}, \star_{2}$ two distinct symbols not in $X$ and $\Delta_{n} X^{\star_{1}, \star_{2}}:=\Delta_{n}(X \cup$ $\left.\left\{\star_{1}, \star_{2}\right\}\right)$. We define a $\left(\star_{1}, \star_{2}\right)$-DRB monomial on $\Delta_{n} X$ to be an expression in $\mathcal{B}\left(\Delta_{n} X^{\star_{1}, \star_{2}}\right)$ with exactly one occurrence of $\star_{1}$ and exactly one occurrence of $\star_{2}$. The set of all $\left(\star_{1}, \star_{2}\right)$-DRB 
monomials on $\Delta_{n} X$ is denoted by $\mathcal{B}^{\star_{1}, \star_{2}}\left(\Delta_{n} X\right)$. For $q \in \mathcal{B}^{\star_{1}, \star_{2}}\left(\Delta_{n} X\right)$ and $u_{1}, u_{2} \in \mathbf{k} \mathcal{B}\left(\Delta_{n} X\right)$, we define

$$
\left.q\right|_{u_{1}, u_{2}}:=\left.q\right|_{\star_{1} \mapsto u_{1}, \star_{2} \mapsto u_{2}}
$$

to be the bracketed monomial obtained by replacing the letter $\star_{1}\left(\right.$ resp. $\left.\star_{2}\right)$ in $q$ by $u_{1}\left(\right.$ resp. $\left.u_{2}\right)$ and call it a $\left(u_{1}, u_{2}\right)$-bracketed monomial on $\Delta_{n} X$.

A $\left(u_{1}, u_{2}\right)$-DRB monomial on $\Delta_{n} X$ can also be recursively defined by

$$
\left.q\right|_{u_{1}, u_{2}}:=\left.\left(\left.q^{\star_{1}}\right|_{u_{1}}\right)\right|_{u_{2}}
$$

where $q^{\star_{1}}$ is $q$ when $q$ is regarded as a $\star_{1}$-DRB monomial on the set $\Delta_{n} X^{\star_{2}}$. Then $\left.q^{\star_{1}}\right|_{u_{1}}$ is in $\mathcal{B}^{\star_{2}}\left(\Delta_{n} X\right)$. Similarly, we have

$$
\left.q\right|_{u_{1}, u_{2}}:=\left.\left(\left.q^{\star_{2}}\right|_{u_{2}}\right)\right|_{u_{1}} .
$$

Let $X$ be a well-ordered set and let $Y=\Delta X$. Let $n \geq 0$ be given. For $x_{0}^{\left(i_{0}\right)}, x_{1}^{\left(i_{1}\right)} \in \Delta X$ (resp. $\left.\Delta_{n} X\right)$ with $x_{0}, x_{1} \in X$, define

$$
x_{0}^{\left(i_{0}\right)} \leq x_{1}^{\left(i_{1}\right)}\left(\operatorname{resp} . x_{0}^{\left(i_{0}\right)} \leq_{n} x_{1}^{\left(i_{1}\right)}\right) \Leftrightarrow\left(x_{0},-i_{0}\right) \leq\left(x_{1},-i_{1}\right) \quad \text { lexicographically. }
$$

For example $x^{(2)}<x^{(1)}<x$. Also, $x_{1}<x_{2}$ implies $x_{1}^{(2)}<x_{2}^{(2)}$. Then by [1], the order $\leq_{n}$ is a well order on $\Delta_{n} X$ and hence is extended to a well order on $C\left(\Delta_{n} X\right)$ by Eq. (19) which we still denote by $\leq_{n}$.

We next extend the well order $\leq_{n}$ on $C\left(\Delta_{n} X\right)$ defined above to $\mathcal{B}\left(\Delta_{n} X\right)$. Note that

$$
\mathcal{B}\left(\Delta_{n} X\right)=\left\{u_{0} \otimes u_{1} \otimes \cdots u_{k} \mid u_{i} \in C\left(\Delta_{n} X\right), 1 \leq i \leq k, k \geq 0\right\}=\sqcup_{k \geq 1} C\left(\Delta_{n} X\right)^{\otimes k}
$$

can be identified with the free semigroup on the set $C\left(\Delta_{n} X\right)$. Thus the well order $\leq_{n}$ on $C\left(\Delta_{n} X\right)$ extends to a well order $\leq_{n, \text { lex }}^{*}[1]$ which we will still denote by $\leq_{n}$ for simplicity. More precisely, for any $u=u_{0} \otimes \cdots \otimes u_{k} \in C\left(\Delta_{n} X\right)^{\otimes(k+1)}$ and $v=v_{0} \otimes \cdots \otimes v_{\ell} \in C\left(\Delta_{n} X\right)^{\otimes(\ell+1)}$, define

$$
u \leq_{n} v \text { if }\left(k+1, u_{0}, \cdots, u_{k}\right) \leq\left(\ell+1, v_{0}, \cdots, v_{\ell}\right) \text { lexicographically. }
$$

This is the order on $\mathfrak{B}\left(\Delta_{n} X\right)$ that we will consider in this paper.

Definition 3.7. Let $\leq_{n}$ be the well order on $\mathfrak{B}\left(\Delta_{n} X\right)$ defined in Eq. (26). Let $q \in \mathcal{B}^{\star}\left(\Delta_{n} X\right)$ and $s \in \mathbf{k} \mathcal{B}\left(\Delta_{n} X\right)$.

(a) For any $0 \neq f \in \mathbf{k} \mathcal{B}\left(\Delta_{n} X\right)$, let $\bar{f}$ denote the leading term of $f: f=c \bar{f}+\sum_{i} c_{i} u_{i}$, where $0 \neq c, c_{i} \in \mathbf{k}, u_{i} \in \mathcal{B}\left(\Delta_{n} X\right), u_{i}<\bar{f} . f$ is called monic if $c=1$.

(b) Denote

$$
\left.\overline{\left.q\right|_{s}}:=\overline{\operatorname{Red}\left(\left.q\right|_{s}\right.}\right),
$$

where Red : $\mathbf{k} \mathfrak{C}\left(\Delta_{n} X\right) \rightarrow \amalg\left(\Delta_{n} X\right)=\mathbf{k} \mathcal{B}\left(\Delta_{n} X\right)$ is the reduction map in Eq. (18).

(c) The element $\left.q\right|_{s} \in \mathbf{k} \mathfrak{C}\left(\Delta_{n} X\right)$ is called normal if $\left.q\right|_{s}$ is in $\mathcal{B}\left(\Delta_{n} X\right)$. In other words, if $\operatorname{Red}\left(\left.q\right|_{\bar{s}}\right)=\left.q\right|_{\bar{s}}$.

Remark 3.8. (a) By definition, $\left.q\right|_{s}$ is normal if and only if $\left.q\right|_{s}$ is normal if and only if the $\bar{s}$-DRB monomial $\left.q\right|_{s}$ is already a DRB monomial, that is, no further reduction in $\amalg\left(\Delta_{n} X\right)$ is possible.

(b) Examples of not normal (abnormal) $s$-DRB monomials are

(i) $q=\star P(x)$ and $\bar{s}=P(x)$, giving $\left.q\right|_{s}=P(x) P(x)$ which is reduced to $P(x P(y))+$ $P(P(x) y)+\lambda P(x y)$ in $\amalg\left(\Delta_{n} X\right)$

(ii) $q=d(\star)$ and $\bar{s}=P(x)$, giving $\left.q\right|_{\bar{s}}=d(P(x))$ which is reduced to $x$ in $\amalg\left(\Delta_{n} X\right)$; 
(iii) $q=d(\star)$ and $\bar{s}=x^{2}$, giving $\left.q\right|_{\bar{s}}=d\left(x^{2}\right)$ which is reduced to $2 x x^{(1)}+\lambda\left(x^{(1)}\right)^{2}$ in $\mathrm{U}\left(\Delta_{n} X\right)$

(iv) $q=d^{n}(\star)$ and $\bar{s}=d(x)$, giving $\left.q\right|_{\bar{s}}=d^{n+1}(s)$ which is reduced to 0 in $\amalg\left(\Delta_{n} X\right)$.

Definition 3.9. A weakly monomial order on $\mathcal{B}\left(\Delta_{n} X\right)$ is a well order $\geq$ satisfying the following condition:

for $u, v \in \mathcal{B}\left(\Delta_{n} X\right), u>v \Rightarrow \overline{\left.q\right|_{u}}>\overline{\left.q\right|_{v}}$ if either $q \in \mathcal{B}_{I I}^{\star}\left(\Delta_{n} X\right)$, or $q \in \mathcal{B}_{I}^{\star}\left(\Delta_{n} X\right)$ and $\left.q\right|_{u}$ is normal.

We shall prove that the order defined in Eq. (26) is a weakly monomial order on $\mathcal{B}\left(\Delta_{n} X\right)$. We need the following lemmas.

Lemma 3.10. Let $\ell \geq 1$ and $s \in \mathcal{B}\left(\Delta_{n} X\right)$. Then $\left.d^{\ell}(\star)\right|_{s}$ is normal if and only if $s \in \Delta_{n-\ell} X$.

Proof. If $\bar{s} \in \Delta_{n-\ell} X$, then $d^{\ell}(\bar{s})$ is in $\Delta_{n} X$ and hence $\left.d^{\ell}(\star)\right|_{s}$ is normal. Conversely, if $\bar{s} \notin \Delta_{n-\ell} X$, then either $\operatorname{dep}(\bar{s}) \geq 2$, or $\operatorname{dep}(\bar{s})=1$ and $\operatorname{deg}_{\Delta_{n} X}(u) \geq 2$, or $\bar{s} \in \Delta_{n} X \backslash \Delta_{n-\ell} X$. In all these cases, $\left.d^{\ell}(\star)\right|_{s}$ is not normal.

Lemma 3.11. Let $\leq_{n}$ be the order defined in Eq. (26). Let $u, v \in \mathcal{B}\left(\Delta_{n} X\right)$ and $\ell \in \mathbb{Z}_{\geq 1}$. If $u>_{n} v$ and $\left.d^{\ell}(\star)\right|_{u}$ is normal, then $\overline{d^{\ell}(u)}>_{n} \overline{d^{\ell}(v)}$.

Proof. We prove the result by induction on $\ell$. We first consider $\ell=1$ and prove $\overline{d(u)}>_{n} \overline{d(v)}$. Since $\left.d(\star)\right|_{u}$ is normal, we have $u=x_{1}^{\left(i_{1}\right)} \in \Delta_{n-1} X$ by Lemma 3.10. Since $u>_{n} v$, by the definition of $>_{n}$, we have we have $v=x_{2}^{\left(i_{2}\right)} \in \Delta_{n} X$ with either $x_{1}>x_{2}$ or $x_{1}=x_{2}$ and $i_{1}<i_{2}$. So $\overline{d(u)}>_{n} \overline{d(v)}$.

Next, suppose the result holds for $1 \leq m<\ell$. Then by the induction hypothesis, we have

$$
\overline{d^{\ell}(u)}=\overline{d\left(d^{\ell-1}(u)\right)}=\overline{d\left(\overline{d^{\ell-1}(u)}\right)}>_{n} \overline{\left.d \overline{\left(d^{\ell-1}(v)\right.}\right)}=\overline{d\left(d^{\ell-1}(v)\right)}=\overline{d^{\ell}(v)} .
$$

Proposition 3.12. The order $\leq_{n}$ defined in Eq. (26) is a weakly monomial order.

Proof. Let $u, v \in \mathcal{B}\left(\Delta_{n} X\right)$ with $u>_{n} v$ and $q \in \mathcal{B}^{\star}\left(\Delta_{n} X\right)$. By Lemma 3.5 we have the following three cases to consider.

Case 1. Consider $q=s \star t$ where $s \in C\left(\Delta_{n} X\right)$ and $t \in \mathcal{B}\left(\Delta_{n} X\right)$. Note that $\mathcal{B}\left(\Delta_{n} X\right)=C\left(\Delta_{n} X\right) \sqcup$ $C(\Delta X) P\left(\mathcal{B}\left(\Delta_{n} X\right)\right)$. We consider the following four subcases depending on $t$ or $u$ in $C\left(\Delta_{n} X\right)$ or $C(\Delta X) P\left(\mathcal{B}\left(\Delta_{n} X\right)\right)$.

Subcase 1.1. Let $t, u \in C\left(\Delta_{n} X\right)$. Since $u>_{n} v$, we have that $v \in C\left(\Delta_{n} X\right)$ and so by Lemma 3.1, $\overline{\left.q\right|_{u}}=s u t>_{n} s v t=\overline{\left.q\right|_{v}}$.

Subcase 1.2. Let $t \in C\left(\Delta_{n} X\right) P\left(\mathcal{B}\left(\Delta_{n} X\right)\right)$ and $u \in C\left(\Delta_{n} X\right)$. Let $t=t_{0} \otimes \cdots \otimes t_{m}$ with $m \geq 1$. Since $u>_{n} v$, we have $v \in C\left(\Delta_{n} X\right)$. By Lemma 3.1 and Eq. (26), we have

$$
\left(m+1, s u t_{0}, t_{1}, \cdots, t_{m}\right)>\left(m+1, s v t_{0}, t_{1}, \cdots, t_{m}\right) \quad \text { lexicographically. }
$$

So $\overline{\left.q\right|_{u}}=\left(s u t_{0}\right) \otimes t_{1} \otimes \cdots \otimes t_{m}>_{n}\left(s v t_{0}\right) \otimes t_{1} \otimes \cdots \otimes t_{m}=\overline{\left.q\right|_{v}}$.

Subcase 1.3. Let $t \in C\left(\Delta_{n} X\right)$ and $u \in C\left(\Delta_{n} X\right) P\left(\mathcal{B}\left(\Delta_{n} X\right)\right)$. Let $u=u_{0} \otimes \cdots \otimes u_{k}$ with $k \geq 1$. If $v \in C\left(\Delta_{n} X\right)$, it is obvious that

$$
\overline{\left.q\right|_{u}}=\left(s t u_{0}\right) \otimes \cdots \otimes u_{k}>_{n} \overline{\left.q\right|_{v}}=s v t .
$$

If $v \in C\left(\Delta_{n} X\right) P\left(\mathcal{B}\left(\Delta_{n} X\right)\right)$, let $v=v_{0} \otimes \cdots \otimes v_{m}$ with $m \geq 1$. Then $\overline{\left.q\right|_{v}}=\left(s t v_{0}\right) \otimes \cdots \otimes v_{m}$. Since $u>_{n} v$, by Eq. (26), we have that

$$
\left(k+1, u_{0}, \cdots, u_{k}\right)>\left(m+1, v_{0}, \cdots, v_{m}\right) \quad \text { lexicographically. }
$$


By Lemma 3.1, it follows that

$$
\left(k+1, s t u_{0}, u_{1}, \cdots, u_{k}\right)>\left(m+1, \text { st } v_{0}, v_{1}, \cdots, v_{m}\right) \quad \text { lexicographically, }
$$

that is, $\overline{\left.q\right|_{u}}>_{n} \overline{\left.q\right|_{v}}$.

Subcase 1.4. Let $t, u \in C\left(\Delta_{n} X\right) P\left(\mathcal{B}\left(\Delta_{n} X\right)\right)$. Let $t=t_{1} \otimes \tilde{t}=t_{0} P(\tilde{t})$ and $u=u_{0} \otimes \tilde{u}=u_{0} P(\tilde{u})$, where $t_{0}, u_{0} \in C\left(\Delta_{n} X\right), \tilde{t}, \tilde{u} \in \mathcal{B}\left(\Delta_{n} X\right)$. If $v \in C\left(\Delta_{n} X\right)$, then

$$
\overline{\left.q\right|_{v}}=\left(s v t_{0}\right) P(\tilde{t}) \quad \text { and } \quad \overline{\left.q\right|_{u}}=\overline{s t_{0} u_{0} P(\tilde{t}) P(\tilde{u})}=s t_{0} u_{0} \overline{P(\tilde{t}) P(\tilde{u})} .
$$

Thus $\operatorname{dep}\left(\overline{\left.q\right|_{u}}\right)>\operatorname{dep}\left(\overline{\left.q\right|_{v}}\right)$ and so $\overline{\left.q\right|_{u}}>_{n} \overline{\left.q\right|_{v}}$. If $v \in C(\Delta X) P\left(\mathcal{B}\left(\Delta_{n} X\right)\right)$, let $v=v_{0} \otimes \tilde{v}=v_{0} P(\tilde{v})$. Then $\overline{\left.q\right|_{v}}=\overline{s t_{0} v_{0} P(\tilde{t}) P(\tilde{v})}=s t_{0} v_{0} \overline{P(\tilde{t}) P(\tilde{v})}$. Since $u>_{n} v$, we have $\operatorname{dep}(\tilde{u})+1=\operatorname{dep}(u) \geq \operatorname{dep}(v)=$ $\operatorname{dep}(\tilde{v})+1$ by Eq. (26) and $\operatorname{sodep}(\tilde{u}) \geq \operatorname{dep}(\tilde{v}) . \operatorname{If} \operatorname{dep}(\tilde{u})>\operatorname{dep}(\tilde{v})$, then $\operatorname{dep}\left(\overline{\left.q\right|_{u}}\right)>\operatorname{dep}\left(\overline{\left.q\right|_{v}}\right)$ and so $\overline{\left.q\right|_{u}}>_{n} \overline{\left.q\right|_{v}}$. Suppose $\operatorname{dep}(\tilde{u})=\operatorname{dep}(\tilde{v})$. Then $\operatorname{dep}\left(\overline{\left.q\right|_{u}}\right)=\operatorname{dep}\left(\overline{\left.q\right|_{v}}\right)$. If $u_{0}>_{n} v_{0}$, then $s t_{0} u_{0}>_{n} s t_{0} v_{0}$ by Lemma 3.1 and so $\overline{\left.q\right|_{u}}>_{n} \overline{\left.q\right|_{v}}$ by Eq. (26). We are left to consider the case $\operatorname{dep}(\tilde{u})=\operatorname{dep}(\tilde{v})$ and $u_{0}=v_{0}$. In this case, since $u>_{n} v$, we have that $\tilde{u}>_{n} \tilde{v}$. If $\tilde{u}>_{n} \tilde{v} \geq \tilde{t}$, then

$$
\begin{gathered}
\overline{\left.q\right|_{u}}=\left(s t_{0} u_{0}\right) \overline{P(\tilde{t}) P(\tilde{u})}=\left(s t_{0} u_{0}\right) P(\tilde{u} P(\tilde{t}))=\left(s t_{0} u_{0}\right) \otimes \tilde{u} \otimes \tilde{t} \\
>_{n}\left(s t_{0} v_{0}\right) \otimes \tilde{v} \otimes \tilde{t}=\left(s t_{0} v_{0}\right) P(\tilde{v} P(\tilde{t}))=\left(s t_{0} v_{0}\right) \overline{P(\tilde{t}) P(\tilde{v})}=\overline{\left.q\right|_{v}} .
\end{gathered}
$$

If $\tilde{t} \geq \tilde{u}>_{n} \tilde{v}$, then

$$
\begin{gathered}
\overline{\left.q\right|_{u}}=\left(s t_{0} u_{0}\right) \overline{P(\tilde{t}) P(\tilde{u})}=\left(s t_{0} u_{0}\right) P(\tilde{t} P(\tilde{u}))=\left(s t_{0} u_{0}\right) \otimes \tilde{t} \otimes \tilde{u} \\
>_{n}\left(s t_{0} v_{0}\right) \otimes \tilde{t} \otimes \tilde{v}=\left(s t_{0} u_{0}\right) P(\tilde{t} P(\tilde{v}))=\left(s t_{0} u_{0}\right) \overline{P(\tilde{t}) P(\tilde{v})}=\overline{\left.q\right|_{v}} .
\end{gathered}
$$

If $\tilde{u}>_{n} \tilde{t}>_{n} \tilde{v}$, then

$$
\begin{gathered}
\overline{\left.q\right|_{u}}=\left(s t_{0} u_{0}\right) \overline{P(\tilde{t}) P(\tilde{u})}=\left(s t_{0} u_{0}\right) P(\tilde{u} P(\tilde{t}))=\left(s t_{0} u_{0}\right) \otimes \tilde{u} \otimes \tilde{t} \\
>_{n}\left(s t_{0} v_{0}\right) \otimes \tilde{t} \otimes \tilde{v}=\left(s t_{0} u_{0}\right) P(\tilde{t} P(\tilde{v}))=\left(s t_{0} u_{0}\right) \overline{P(\tilde{t}) P(\tilde{v})}=\overline{\left.q\right|_{v}} .
\end{gathered}
$$

Case 2. Consider $q=s P(p)$ for some $s \in C\left(\Delta_{n} X\right)$ and $p \in \mathcal{B}^{\star}\left(\Delta_{n} X\right)$. This case can be verified by induction on $\operatorname{dep}(q)$ and the fact that, for $u, v \in \mathcal{B}\left(\Delta_{n} X\right), u>_{n} v$ implies $P(u)>_{n} P(v)$.

Case 3. Consider $q \in \mathcal{B}_{I}^{\star}\left(\Delta_{n} X\right)$. Then $q=\left.p\right|_{d^{\ell}(\star)}$ for some $p \in \mathcal{B}^{\star}\left(\Delta_{n} X\right)$ and $\ell \in \mathbb{Z}_{\geq 1}$. Take such $\ell$ maximal so that $p \in \mathcal{B}_{I I}^{\star}\left(\Delta_{n} X\right)$. We need to show that if $u>_{n} v$ and $\left.q\right|_{u}$ is normal, then $\overline{\left.q\right|_{u}}>_{n} \overline{\left.q\right|_{v}}$. But if $\left.q\right|_{u}$ is normal then $\left.d^{\ell}(\star)\right|_{u}$ is normal. Then by Lemma 3.11, we have $\overline{d^{\ell}(u)}>_{n} \overline{d^{\ell}(v)}$. Then by Cases 1 and 2, we have $\overline{\left.q\right|_{u}}=\overline{\left.p\right|_{d^{\ell}(u)}}>_{n} \overline{\left.p\right|_{d^{\ell}(v)}}=\overline{\left.q\right|_{v}}$. This completes the proof.

We give the following consequences of Proposition 3.12 to be applied in Section 4.

Lemma 3.13. Let $q \in \mathcal{B}^{\star}\left(\Delta_{n} X\right)$ and $s \in \mathbf{k} \mathcal{B}\left(\Delta_{n} X\right)$ be monic. If $\left.q\right|_{s}$ is normal, then $\overline{\left.q\right|_{s}}=\left.q\right|_{\bar{s}}$.

Proof. Let $s=\bar{s}+\sum_{i} c_{i} s_{i}$ with $0 \neq c_{i} \in \mathbf{k}$ and $s_{i}<\bar{s}$. Then $\left.q\right|_{s}=\left.q\right|_{s}+\left.\sum_{i} c_{i} q\right|_{s_{i}}$. Since $\left.q\right|_{s}$ is normal, it follows that $\left.q\right|_{\bar{s}} \in \mathcal{B}\left(\Delta_{n} X\right)$ and so $\overline{\left.q\right|_{s}}=\left.q\right|_{\bar{s}}$. We have the following two cases to consider.

Case I. $q \in \mathcal{B}_{I I}^{\star}\left(\Delta_{n} X\right)$. Then $\overline{\left.q\right|_{s_{i}}}<\overline{\left.q\right|_{s}}=\left.q\right|_{\bar{s}}$ by Definition 3.9 and Proposition 3.12. Hence $\overline{\left.q\right|_{s}}=\overline{\left.q\right|_{s}}=\left.q\right|_{\bar{s}}$.

Case II. $q \in \mathcal{B}_{I}^{\star}\left(\Delta_{n} X\right)$. Then $q=\left.p\right|_{d^{\ell}(\star)}$ for some $p \in \mathcal{B}^{\star}\left(\Delta_{n} X\right)$ and $\ell \in \mathbb{Z}_{\geq 1}$. Since $\left.q\right|_{s}=\left.p\right|_{d^{\ell}(s)}$ is normal, we have $\bar{s} \in \Delta_{n-\ell} X$ by Lemma 3.10. Furthermore, $s_{i}<\bar{s}$ implies that $s_{i} \in \Delta_{n} X$. Thus by Definition 3.9 and Proposition 3.12, we have $\overline{\left.q\right|_{s_{i}}}<\left.q\right|_{\bar{s}}$. So $\overline{\left.q\right|_{s}}=\left.q\right|_{\bar{s}}$.

Lemma 3.14. Let $u, v \in \mathcal{B}\left(\Delta_{n} X\right)$ with $u>v$ and $q \in \mathcal{B}^{\star}\left(\Delta_{n} X\right)$. If $\left.q\right|_{u}$ is normal, then either $\left.q\right|_{v}=0$ or $\left.q\right|_{v}$ is also normal. 
Proof. Suppose that $\left.q\right|_{v}$ is not normal. Then $\left.q\right|_{v} \notin \mathcal{B}\left(\Delta_{n} X\right)$. We have the following cases to consider.

Case I. $\operatorname{dep}(v) \geq 2$, that is, $v \in C\left(\Delta_{n} X\right) P\left(\mathcal{B}\left(\Delta_{n} X\right)\right)$, and $q=\left.p\right|_{\star P(w)}$ for some $p \in \mathcal{B}^{\star}\left(\Delta_{n} X\right)$ and $w \in \mathcal{B}\left(\Delta_{n} X\right)$. Since $u>v$, it follows that $\operatorname{dep}(u) \geq \operatorname{dep}(v) \geq 2$ and so $u \in C\left(\Delta_{n} X\right) P\left(\mathcal{B}\left(\Delta_{n} X\right)\right)$. This implies that $\left.q\right|_{u}$ can be reduced by the Rota-Baxter relation and so $\left.q\right|_{u} \notin \mathcal{B}\left(\Delta_{n} X\right)$. Hence $\left.q\right|_{u}$ is not normal, a contradiction.

Case II. $q=\left.p\right|_{d^{\ell}(\star)}$ for some $p \in \mathcal{B}^{\star}\left(\Delta_{n} X\right)$ and $\ell \geq 1$. If $\operatorname{dep}(v) \geq 2$, then since $u>v$, we have $\operatorname{dep}(u) \geq 2$ and so $u \in C\left(\Delta_{n} X\right) P\left(\mathcal{B}\left(\Delta_{n} X\right)\right)$. This implies that $\left.q\right|_{u}$ is not normal, a contradiction. If $\operatorname{dep}(v)=1$, then $v \in C\left(\Delta_{n} X\right)$. If further $\operatorname{deg}_{\Delta_{n} X}(v) \geq 2$, then since $u>v$, we have either $\operatorname{dep}(u) \geq 2$, or $\operatorname{dep}(u)=1$ and $\operatorname{deg}_{\Delta_{n} X}(u) \geq 2$. In either case, we have that $\left.q\right|_{u}$ is not normal, a contradiction. Thus we must have $\operatorname{dep}(v)=1$ and $\operatorname{deg}_{\Delta_{n} X}(v)=1$. So $v=x^{(r)}, r \geq 1$. Since $\left.q\right|_{v}=\left.p\right|_{d^{\ell}(v)}=\left.p\right|_{x^{(\ell+r)}}$ is supposed to be not normal, we have $\ell+r>n$. That is, $\left.q\right|_{v}=\left.p\right|_{d^{\ell}(v)}=0$.

\section{Composition-Diamond Lemma}

In this section, we shall establish the composition-diamond lemma for the order $n$ free commutative differential Rota-Baxter algebra $\amalg\left(\mathbf{k}\left[\Delta_{n} X\right]\right)$.

Definition 4.1. (a) Let $u, w \in \mathcal{B}\left(\Delta_{n} X\right)$. We call $u$ a subword of $w$ if $w$ is in the operated ideal of $\mathfrak{C}\left(\Delta_{n} X\right)$ generated by $u$. In terms of $\star$-words, $u$ is a subword of $w$ if there is a $q \in \mathcal{B}^{\star}\left(\Delta_{n} X\right)$ such that $w=\left.q\right|_{u}$.

(b) Let $u_{1}$ and $u_{2}$ be two subwords of $w . u_{1}$ and $u_{2}$ are called separated if $u_{1} \in C\left(\Delta_{n} X\right)$, $u_{2} \in \mathcal{B}\left(\Delta_{n} X\right)$ and there is a $q \in \mathcal{B}^{\star_{1}, \star_{2}}\left(\Delta_{n} X\right)$ such that $w=\left.q\right|_{u_{1}, u_{2}}$.

(c) For any $u \in \mathcal{B}\left(\Delta_{n} X\right), u$ can be expressed as $u=u_{1} \cdots u_{k}$, where $u_{1}, \cdots, u_{k-1} \in \Delta_{n} X$ and $u_{k} \in \Delta_{n} X \cup P\left(\mathcal{B}\left(\Delta_{n} X\right)\right)$. The integer $k$ is called the breath of $u$ and is denoted by bre $(u)$.

(d) Let $f, g \in \mathcal{B}\left(\Delta_{n} X\right)$. A pair $(u, v)$ with $u \in \mathcal{B}\left(\Delta_{n} X\right)$ and $v \in C\left(\Delta_{n} X\right)$ is called an intersection pair for $(f, g)$ if the differential Rota-Baxter monomial $w:=f u$ equals $v g$ and satisfies $\operatorname{bre}(w)<\operatorname{bre}(f)+\operatorname{bre}(g)$. Then we call $f$ and $g$ to be overlapping. Note that if $f$ and $g$ are overlapping, then $f \in C\left(\Delta_{n} X\right)$.

There are four kinds of compositions.

Definition 4.2. Let $\leq$ be a weakly monomial order on $\mathcal{B}\left(\Delta_{n} X\right)$ and $f, g \in \mathbf{k} \mathcal{B}\left(\Delta_{n} X\right)$ monic with respect to $\leq$.

(a) If $\bar{f} \in C\left(\Delta_{n} X\right) P\left(\mathcal{B}\left(\Delta_{n} X\right)\right)$, then define a composition of (right) multiplication to be $f u$ where $u \in C\left(\Delta_{n} X\right) P\left(\mathcal{B}\left(\Delta_{n} X\right)\right)$.

(b) If $\bar{f} \notin \Delta_{n} X$, then define a composition of derivation to be $d^{\ell}(f)$, where $\ell \in \mathbb{Z}_{\geq 1}$.

(c) If there is an intersection pair $(u, v)$ for $(\bar{f}, \bar{g})$, then we define

$$
(f, g)_{w}:=(f, g)_{w}^{u, v}:=f u-v g
$$

and call it an intersection composition of $f$ and $g$.

(d) If there exists a $q \in \mathcal{B}^{\star}\left(\Delta_{n} X\right)$ such that $w:=\bar{f}=\left.q\right|_{\bar{g}}$, then we define $(f, g)_{w}:=(f, g)_{w}^{q}:=$ $f-\left.q\right|_{g}$ and call it an including composition of $f$ and $g$ with respect to $q$. Note that if this is the case, then $\left.q\right|_{g}$ is normal.

In the last two cases, $(f, g)_{w}$ is called the ambiguity of the composition.

Definition 4.3. Let $\leq$ be a weakly monomial order on $\mathcal{B}\left(\Delta_{n} X\right), S \subseteq \mathbf{k} \mathcal{B}\left(\Delta_{n} X\right)$ be a set of monic differential Rota-Baxter polynomials and $w \in \mathcal{B}\left(\Delta_{n} X\right)$. 
(a) An element $h \in \mathbf{k} \mathfrak{B}\left(\Delta_{n} X\right)$ is called trivial $\bmod [S]$ and denote this by

$$
h \equiv 0 \bmod [S]
$$

if $h=\left.\sum_{i} c_{i} q_{i}\right|_{s_{i}}$, where $c_{i} \in \mathbf{k}, q_{i} \in \mathcal{B}^{\star}\left(\Delta_{n} X\right), s_{i} \in S,\left.q_{i}\right|_{s_{i}}$ is normal and $\left.q_{i}\right|_{\overline{s_{i}}} \leq \bar{h}$. This applies in particular to a composition of multiplication $f u$ and a composition of derivation $d^{\ell}(f)$ where $f \in \mathbf{k} \mathfrak{B}\left(\Delta_{n} X\right), u \in P\left(\mathfrak{B}\left(\Delta_{n} X\right)\right)$ and $\ell \geq 1$. We use $\bmod [S]$ to distinguish from the usual notion of $u \equiv 0 \bmod (S)$ when $u$ is in the ideal generated by $S$.

(b) For $u, v \in \mathbf{k} \mathcal{B}\left(\Delta_{n} X\right)$, we call $u$ and $v$ congruent modulo $(S, w)$ and denote this by

$$
u \equiv v \bmod (S, w)
$$

if $u-v=\left.\sum_{i} c_{i} q_{i}\right|_{s_{i}}$, where $c_{i} \in \mathbf{k}, q_{i} \in \mathcal{B}^{\star}\left(\Delta_{n} X\right), s_{i} \in S,\left.q_{i}\right|_{s_{i}}$ is normal and $\overline{\left.q_{i}\right|_{s_{i}}}<w$.

(c) For $f, g \in \mathbf{k} \mathcal{B}\left(\Delta_{n} X\right)$ and suitable $u, v$ or $q$ that give an intersection composition $(f, g)_{w}^{u, v}$ or an including composition $(f, g)_{w}^{q}$, the composition is called trivial modulo $(S, w)$ if

$$
(f, g)_{w}^{u, v} \text { or }(f, g)_{w}^{q} \equiv 0 \bmod (S, w) .
$$

(d) The set $S \subseteq \mathbf{k} \mathcal{B}\left(\Delta_{n} X\right)$ is a Gröbner-Shirshov basis if all compositions of multiplication and derivation are trivial mod $[S]$, and, for $f, g \in S$, all intersection compositions $(f, g)_{w}^{u, v}$ and all including compositions $(f, g)_{w}^{q}$ are trivial modulo $(S, w)$.

We give some preparational lemmas before establishing the Composition-Diamond Lemma.

Lemma 4.4. Let $\geq$ be the weakly monomial order on $\mathcal{B}\left(\Delta_{n} X\right)$ defined in Eq. (26), $s_{1}, s_{2} \in$ $\mathbf{k} \mathcal{B}\left(\Delta_{n} X\right), q_{1}, q_{2} \in \mathcal{B}^{\star}\left(\Delta_{n} X\right)$ and $w \in \mathcal{B}\left(\Delta_{n} X\right)$ such that $w=\left.q_{1}\right|_{s_{1}}=\left.q_{2}\right|_{\overline{s_{2}}}$, where each $\left.q_{i}\right|_{s_{i}}$ is normal, $i=1,2$. If $\overline{s_{1}}$ and $\overline{s_{2}}$ are separated in $w$, then $\left.\left.q_{1}\right|_{s_{1}} \equiv q_{2}\right|_{s_{2}} \bmod (S, w)$.

Proof. Let $q \in \mathcal{B}^{\star_{1}, \star_{2}}\left(\Delta_{n} X\right)$ be the $\left(\star_{1}, \star_{2}\right)$-DRB monomial obtained by replacing this occurrence of $\overline{s_{1}}$ in $w$ by $\star_{1}$ and this occurrence of $\overline{s_{2}}$ in $w$ by $\star_{2}$. Then we have

$$
\left.q^{\star}\right|_{\overline{s_{1}}}=q_{2},\left.q^{\star_{2}}\right|_{\overline{s_{2}}}=q_{1} \text { and }\left.q\right|_{\overline{s_{1}}, \overline{s_{2}}}=\left.q_{1}\right|_{\overline{s_{1}}}=\left.q_{2}\right|_{\overline{s_{2}}} \text {, }
$$

where in the first two equalities, we have identified $\mathcal{B}^{\star_{2}}\left(\Delta_{n} X\right)$ and $\mathcal{B}^{\star_{1}}\left(\Delta_{n} X\right)$ with $\mathcal{B}^{\star}\left(\Delta_{n} X\right)$. Let $s_{1}-\overline{s_{1}}=\sum_{i} c_{i} u_{i}$ and $s_{2}-\overline{s_{2}}=\sum_{j} d_{j} v_{j}$ with $c_{i}, d_{j} \in \mathbf{k}$ and $u_{i}, v_{j} \in \mathcal{B}\left(\Delta_{n} X\right)$. Then by the linearity of $s_{1}$ and $s_{2}$ in $\left.q\right|_{s_{1}, s_{2}}$, we have

$$
\begin{aligned}
\left.q_{1}\right|_{s_{1}}-\left.q_{2}\right|_{s_{2}} & =\left.\left(q^{\star_{2}} \mid \overline{s_{2}}\right)\right|_{s_{1}}-\left.\left(q^{\star_{1}} \mid \overline{s_{1}}\right)\right|_{s_{2}} \\
& =\left.q\right|_{s_{1}, \overline{s_{2}}}-\left.q\right|_{\overline{s_{1}}, s_{2}} \\
& =\left.q\right|_{s_{1}, \overline{s_{2}}}-\left.q\right|_{s_{1}, s_{2}}+\left.q\right|_{s_{1}, s_{2}}-\left.q\right|_{\overline{s_{1}}, s_{2}} \\
& =-\left.q\right|_{s_{1}, s_{2}-\overline{s_{2}}}+\left.q\right|_{s_{1}-\overline{s_{1}}, s_{2}} \\
& =-\left.\left(\left.q^{\star_{2}}\right|_{s_{2}-\overline{s_{2}}}\right)\right|_{s_{1}}+\left.\left(\left.q^{\star_{1}}\right|_{s_{1}-\overline{s_{1}}}\right)\right|_{s_{2}} \\
& =-\left.\sum_{j} d_{j}\left(\left.q^{\star_{2}}\right|_{v_{j}}\right)\right|_{s_{1}}+\left.\sum_{i} c_{i}\left(\left.q^{\star_{1}}\right|_{u_{i}}\right)\right|_{s_{2}} \\
& =-\left.\sum_{j} d_{j} q\right|_{s_{1}, v_{j}}+\left.\sum_{i} c_{i} q\right|_{u_{i}, s_{2}} .
\end{aligned}
$$

Since $\left.\left(\left.q^{\star_{1}}\right|_{s_{1}}\right)\right|_{\overline{s_{2}}}=\left.q\right|_{s_{1}, \overline{s_{2}}}=\left.\left(\left.q^{\star_{2}}\right|_{\overline{s_{2}}}\right)\right|_{s_{1}}=\left.q_{1}\right|_{s_{1}}$ is normal and $v_{j}<\overline{s_{2}}$, by Definition 3.9 and Proposition 3.12, we have

$$
\overline{\left.q\right|_{s_{1}, v_{j}}}=\overline{\left.\left(\left.q^{\star}\right|_{s_{1}}\right)\right|_{v_{j}}}<\overline{\left.\left(\left.q^{\star}\right|_{s_{1}}\right)\right|_{s_{2}}}=\overline{\left.q_{1}\right|_{s_{1}}}=\left.q_{1}\right|_{s_{1}}=w .
$$

Similarly, since $\left.\left(\left.q^{\star_{2}}\right|_{s_{2}}\right)\right|_{s_{1}}=\left.q\right|_{\overline{s_{1}}, s_{2}}=\left.\left(q^{\star}||_{s_{1}}\right)\right|_{s_{2}}=\left.q_{2}\right|_{s_{2}}$ is normal and $u_{i}<\overline{s_{1}}$, we have

$$
\overline{\left.q\right|_{u_{i}, s_{2}}}=\overline{\left.\left(\left.q^{\star 2}\right|_{s_{2}}\right)\right|_{u_{i}}}<\overline{\left.\left(\left.q^{\star 2}\right|_{s_{2}}\right)\right|_{s_{1}}}=\overline{\left.q_{2}\right|_{s_{2}}}=\left.q_{2}\right|_{\overline{s_{2}}}=w \text {. }
$$


Hence $\left.\left.q_{1}\right|_{s_{1}} \equiv q_{2}\right|_{s_{2}} \bmod (S, w)$.

For $q \in \mathcal{B}^{\star}\left(\Delta_{n} X\right)$, let $\operatorname{dep}_{\star}(q)$ be the depth of the symbol $\star$ in $q$. For example, $\operatorname{dep}_{\star}(q)=1$ if $q=P(\star)$ and $\operatorname{dep}_{\star}(q)=2$ if $q=P(x P(\star))$.

Lemma 4.5. Let $\leq_{n}$ be the weakly monomial order on $\mathcal{B}\left(\Delta_{n} X\right)$ defined in Eq. (26) and let $S \subseteq$ $\mathbf{k} \mathcal{B}\left(\Delta_{n} X\right)$. If each composition of multiplication and derivation of $S$ is trivial mod [S], then, for $s \in S$ and $q \in \mathcal{B}^{\star}\left(\Delta_{n} X\right),\left.q\right|_{s}$ is trivial $\bmod [S]$ :

$$
\left.q\right|_{s}=\left.\sum_{i} c_{i} q_{i}\right|_{s_{i}}
$$

where, for each $i, 0 \neq c_{i} \in \mathbf{k}, s_{i} \in S,\left.q_{i}\right|_{s_{i}}$ is normal and $\left.q_{i}\right|_{\overline{s_{i}}} \leq \overline{\left.q\right|_{s}}$.

Proof. We have the following two cases to consider.

Case I. $q \in \mathcal{B}_{I I}^{\star}\left(\Delta_{n} X\right)$. In this case, we prove the result by induction on $\operatorname{dep}_{\star}(q)$. If $\operatorname{dep}_{\star}(q)=0$, then $q=u \star v$, where $u \in C\left(\Delta_{n} X\right)$ and $v \in \mathcal{B}\left(\Delta_{n} X\right)$. If $s \in S$ is such that $\bar{s} \in C\left(\Delta_{n} X\right)$ or $v \in C\left(\Delta_{n} X\right)$, then it is obvious that $\left.q\right|_{s}$ is normal by Definition 3.7 (c). Suppose $\bar{s}, v \notin C\left(\Delta_{n} X\right)$. Then $\bar{s}, v \in C\left(\Delta_{n} X\right) P\left(\mathcal{B}\left(\Delta_{n} X\right)\right)$. Since the composition of multiplication of $S$ is trivial mod $[S]$, we have

$$
s v=\left.\sum_{i} d_{i} p_{i}\right|_{i}
$$

where $0 \neq d_{i} \in \mathbf{k}, t_{i} \in S,\left.p_{i}\right|_{t_{i}}$ is normal and $\left.p_{i}\right|_{t_{i}} \leq \overline{s v}$. Let $q_{i}:=u p_{i} \in \mathcal{B}^{\star}\left(\Delta_{n} X\right)$. Since $u \in C\left(\Delta_{n} X\right)$, we have $\left.q_{i}\right|_{t_{i}}=\left.u p_{i}\right|_{t_{i}}$ is normal. Moreover,

$$
\left.q\right|_{s}=u s v=\left.\sum_{i} d_{i} u p_{i}\right|_{t_{i}}=\left.\sum_{i} d_{i} q_{i}\right|_{t_{i}}
$$

where $\left.q_{i}\right|_{\bar{t}_{i}}=\left.u p_{i}\right|_{\bar{t}_{i}} \leq u \overline{s v}=\overline{u s v}=\overline{\left.q\right|_{s}}$. Hence $\left.q\right|_{s}$ is trivial $\bmod [S]$.

Suppose the claim has been proved for $q \in C\left(\Delta_{n} X\right)$ with $\operatorname{dep}_{\star}(q)=k \geq 0$ and consider $q$ with $\operatorname{dep}_{\star}(q)=k+1$. Then $q=u P(p)$, where $u \in C\left(\Delta_{n} X\right)$ and $p \in \mathcal{B}_{I I}^{\star}\left(\Delta_{n} X\right)$ with $\operatorname{dep}_{\star}(p)=k$. By the induction hypothesis we have $\left.p\right|_{s}=\left.\sum_{i} c_{i} p_{i}\right|_{t_{i}}$, where $0 \neq c_{i} \in \mathbf{k}, t_{i} \in S,\left.p_{i}\right|_{t_{i}}$ is normal and $\left.p_{i}\right|_{\bar{t}_{i}} \leq \overline{\left.p\right|_{s}}$. Let $q_{i}:=u P\left(p_{i}\right) \in \mathcal{B}^{\star}\left(\Delta_{n} X\right)$. Then $\left.q\right|_{s}=\left.\sum_{i} c_{i} q_{i}\right|_{i},\left.q_{i}\right|_{t_{i}}=u P\left(\left.p_{i}\right|_{t_{i}}\right)$ is normal and $\left.q_{i}\right|_{\bar{t}_{i}}=u P\left(\left.p_{i}\right|_{\bar{t}_{i}}\right) \leq u P\left(\overline{\left.p\right|_{s}}\right)=\overline{u P\left(\left.p\right|_{s}\right)}=\overline{\left.q\right|_{s}}$, as desired. This completes the induction.

Case II. $q \in \mathcal{B}_{I}^{\star}\left(\Delta_{n} X\right)$. Then $q=\left.p\right|_{d^{\ell}(\star)}$ for some $p \in \mathcal{B}^{\star}\left(\Delta_{n} X\right)$ and $\ell \geq 1$. Choose such an $\ell$ to be maximal so that $p$ is in $\mathcal{B}_{I I}^{\star}\left(\Delta_{n} X\right)$. By our hypothesis, the composition of derivation is trivial mod $[S]$. So $d^{\ell}(s)=\left.\sum c_{i} p_{i}\right|_{s_{i}}$, where $0 \neq c_{i} \in \mathbf{k}, s_{i} \in S,\left.p_{i}\right|_{s_{i}}$ is normal and $\left.p_{i}\right|_{\bar{s}_{i}} \leq \overline{d^{\ell}(s)}$. Since $p$ is in $\mathcal{B}_{I I}^{\star}\left(\Delta_{n} X\right)$, by Cases I that has been proved above, the result holds.

Lemma 4.6. Let $\geq$ be the weakly monomial order on $\mathcal{B}\left(\Delta_{n} X\right)$ defined in Eq. (26) and let $S \subseteq$ $\mathbf{k} \mathcal{B}\left(\Delta_{n} X\right)$. If $S$ is a Gröbner-Shirshov basis, then for each pair $s_{1}, s_{2} \in S$ for which there exist $q_{1}, q_{2} \in \mathcal{B}^{\star}\left(\Delta_{n} X\right)$ and $w \in \mathcal{B}\left(\Delta_{n} X\right)$ such that $w=\left.q_{1}\right|_{s_{1}}=\left.q_{2}\right|_{\overline{s_{2}}}$ with $\left.q_{1}\right|_{s_{1}}$ and $\left.q_{2}\right|_{s_{2}}$ normal, we have $\left.\left.q_{1}\right|_{s_{1}} \equiv q_{2}\right|_{s_{2}} \bmod (S, w)$.

Proof. Let $s_{1}, s_{2} \in S, q_{1}, q_{2} \in \mathcal{B}^{\star}\left(\Delta_{n} X\right)$ and $w \in \mathcal{B}\left(\Delta_{n} X\right)$ be such that $w=\left.q_{1}\right|_{\overline{s_{1}}}=\left.q_{2}\right|_{\overline{s_{2}}}$. According to the relative location of $s_{1}$ and $s_{2}$ in $w$, we have the following three cases to consider.

Case I. $\overline{s_{1}}$ and $\overline{s_{2}}$ are separated in $w$. This case is covered by Lemma 4.4.

Case II. $\overline{s_{1}}$ and $\overline{s_{2}}$ are overlapping in $w$. Then there are $u \in \mathcal{B}\left(\Delta_{n} X\right)$ and $v \in C(\Delta X)$ such that $w_{1}:=\overline{s_{1}} u=v \overline{s_{2}}$ is a subword in $w$ with $\operatorname{bre}\left(w_{1}\right)<\operatorname{bre}\left(\overline{s_{1}}\right)+\operatorname{bre}\left(\overline{s_{2}}\right)$. Since $S$ is a Gröbner-Shirshov 
basis, we have

$$
s_{1} u-v s_{2}=\left.\sum_{j} c_{j} p_{j}\right|_{t_{j}}
$$

where $\left.p_{j}\right|_{t_{j}}$ is normal and $\overline{\left.p_{j}\right|_{t_{j}}}=\left.p_{j}\right|_{t_{j}}<\overline{s_{1}} u=v \overline{s_{2}}=w_{1}$.

Let $q \in \mathcal{B}^{\star_{1}, \star_{2}}\left(\Delta_{n} X\right)$ be obtained from $q_{1}$ by replacing $\star$ by $\star_{1}$, and the $u$ on the right of $\star$ by $\star_{2}$. Let $p \in \mathcal{B}^{\star}\left(\Delta_{n} X\right)$ be obtained from $q$ by replacing $\star_{1} \star_{2}$ by $\star$. Then we have

$$
\left.q^{\star_{2}}\right|_{u}=q_{1},\left.q^{\star_{1}}\right|_{v}=q_{2} \text { and }\left.p\right|_{\overline{s_{1}} u}=\left.q\right|_{\overline{s_{1}}, u}=\left.q_{1}\right|_{\overline{s_{1}}}=w,
$$

where in the first two equalities, we have identified $\mathcal{B}^{\star_{2}}\left(\Delta_{n} X\right)$ and $\mathcal{B}^{\star_{1}}\left(\Delta_{n} X\right)$ with $\mathcal{B}^{\star}(\Delta X)$. Thus we have

$$
\left.q_{1}\right|_{s_{1}}-\left.q_{2}\right|_{s_{2}}=\left.\left(\left.q^{\star_{2}}\right|_{u}\right)\right|_{s_{1}}-\left.\left(\left.q^{\star_{1}}\right|_{v}\right)\right|_{s_{2}}=\left.p\right|_{s_{1} u-v s_{2}}=\left.\sum_{j} c_{j} p\right|_{\left.p_{j}\right|_{t_{j}}}
$$

Since $\overline{\left.p_{j}\right|_{t_{j}}}<w_{1}$ and $\left.p\right|_{w_{1}}=w \in \mathcal{B}\left(\Delta_{n} X\right)$ is normal, we have $\left.p\right|_{\left.p_{j}\right|_{t_{j}}}$ is either zero or normal by Lemma 3.14. If $\left.p\right|_{\overline{p_{j} \mid t_{j}}}=0$, there is nothing to prove. If $\left.p\right|_{\overline{p_{j} t_{j}}}$ is normal, then by Lemma 3.13, we have $\overline{\left.p\right|_{p_{j} t_{j}}}=\left.p\right|_{p_{j} \mid t_{j}}<\left.p\right|_{w_{1}}=w$. Hence $\left.\left.q_{1}\right|_{s_{1}} \equiv q_{2}\right|_{s_{2}} \bmod (S, w)$.

Case III. One of $\overline{s_{1}}$ or $\overline{s_{2}}$ is a subword of the other. Without loss of generality, we may suppose that $\overline{s_{1}}=\left.q\right|_{\overline{s_{2}}}$ for some $q \in \mathcal{B}^{\star}\left(\Delta_{n} X\right)$. Since $\overline{s_{1}}=\left.q\right|_{\overline{s_{2}}} \in \mathcal{B}\left(\Delta_{n} X\right)$, it follows that $\left.q\right|_{s_{2}}$ is normal by Definition 3.7 and $\overline{\left.q\right|_{s_{2}}}=\left.q\right|_{s_{2}}$. For the inclusion composition $\left(s_{1}, s_{2}\right) \frac{q}{s_{1}}$, since $S$ is a GröbnerShirshov basis, we have $\left(s_{1}, s_{2}\right) \frac{q}{s_{1}}=s_{1}-\left.q\right|_{s_{2}}=\left.\sum_{j} c_{j} p_{j}\right|_{t_{j}}$, where $c_{j} \in \mathbf{k}, p_{j} \in \mathcal{B}^{\star}\left(\Delta_{n} X\right), t_{j} \in S$ and $\left.p_{j}\right|_{t_{j}}$ is normal with $\overline{\left.p_{j}\right|_{t_{j}}}<\overline{s_{1}}$. Let $p \in \mathcal{B}^{\star}\left(\Delta_{n} X\right)$ be obtained from $q_{1}$ by replacing $\star$ with $q$. Then $w=\left.q_{2}\right|_{\overline{s_{2}}}=\left.q_{1}\right|_{\overline{s_{1}}}=\left.q_{1}\right|_{\left.q\right|_{s_{2}}}=\left.p\right|_{\overline{s_{2}}}$. Since $S$ is a Gröbner-Shirshov basis, by Cases I and II, we have

$$
\left.p\right|_{\overline{s_{2}}}-\left.q_{2}\right|_{\overline{s_{2}}}=\left.\sum_{i} d_{i} r_{i}\right|_{v_{i}}
$$

where $d_{i} \in \mathbf{k}, r_{i} \in \mathcal{B}^{\star}\left(\Delta_{n} X\right), v_{i} \in S$ and $\left.r_{i}\right|_{v_{i}}$ is normal with $\overline{\left.r_{i}\right|_{v_{i}}}=\left.r_{i}\right|_{\overline{v_{i}}}<\left.q_{2}\right|_{\overline{s_{2}}}=w$. So

$$
\begin{aligned}
\left.q_{2}\right|_{s_{2}}-\left.q_{1}\right|_{s_{1}} & =\left(\left.p\right|_{s_{2}}-\left.\sum_{i} d_{i} r_{i}\right|_{v_{i}}\right)-\left.q_{1}\right|_{s_{1}} \\
& =\left.p\right|_{s_{2}}-\left.q_{1}\right|_{s_{1}}-\left.\sum_{i} d_{i} r_{i}\right|_{v_{i}} \\
& =\left.q_{1}\right|_{q_{s_{2}}}-\left.q_{1}\right|_{s_{1}}-\left.\sum_{i} d_{i} r_{i}\right|_{v_{i}} \\
& =-\left.q_{1}\right|_{s_{1}-\left.q\right|_{s_{2}}}-\left.\sum_{i} d_{i} r_{i}\right|_{v_{i}} \\
& =-\left.\sum_{j} c_{j} q_{1}\right|_{\left.p_{j}\right|_{j}}-\left.\sum_{i} d_{i} r_{i}\right|_{v_{i}} .
\end{aligned}
$$

Since $\overline{\left.p_{j}\right|_{t_{j}}}<\overline{s_{1}}$ and $\left.q_{1}\right|_{\overline{s_{1}}}=w \in \mathcal{B}\left(\Delta_{n} X\right)$ is normal by our hypothesis, we have $\left.q_{1}\right|_{\overline{p_{j} \mid t_{j}}}=0$ or $\left.q_{1}\right|_{\overline{p_{j} \mid t_{j}}}$ is normal by Lemma 3.14. If $\left.q_{1}\right|_{\overline{p_{j} \mid t_{j}}}=0$, there is noting to prove. If $\left.q_{1}\right|_{\left.p_{j}\right|_{j}}$ is normal, then by Lemma 3.13, $\overline{\left.q_{1}\right|_{p_{j} \mid t_{j}}}=\left.q_{1}\right|_{\overline{p_{j} \mid t_{j}}}<\left.q_{1}\right|_{s_{1}}=w$. Hence $\left.q_{2}\right|_{s_{2}}-\left.q_{1}\right|_{s_{1}} \equiv 0 \bmod (S, w)$. 
Lemma 4.7. Let $\leq_{n}$ be the weakly monomial order on $\mathcal{B}\left(\Delta_{n} X\right)$ defined in Eq. (26), $S \subseteq \mathbf{k} \mathcal{B}\left(\Delta_{n} X\right)$ and $\operatorname{Irr}(\mathrm{S}):=\mathcal{B}\left(\Delta_{n} X\right) \backslash\left\{\left.q\right|_{s}\left|q \in \mathcal{B}^{\star}\left(\Delta_{n} X\right), s \in S, q\right|_{s}\right.$ is normal $\}$. Then for any $f \in \mathbf{k} \mathcal{B}\left(\Delta_{n} X\right), f$ has an expression

$$
f=\sum_{i} c_{i} u_{i}+\left.\sum_{j} d_{j} q_{j}\right|_{s_{j}}
$$

where $0 \neq c_{i}, d_{j} \in \mathbf{k}, u_{i} \in \operatorname{Irr}(\mathrm{S}), \overline{u_{i}} \leq \bar{f}, s_{j} \in S,\left.q_{j}\right|_{s_{j}}$ is normal and $\left.q_{j}\right|_{\overline{j_{j}}} \leq \bar{f}$.

Proof. Suppose the lemma does not hold and let $f$ be a counterexample with minimal $\bar{f}$. Write $f=\sum_{i} c_{i} u_{i}$ where $0 \neq c_{i} \in \mathbf{k}, u_{i} \in \mathcal{B}\left(\Delta_{n} X\right)$ and $u_{1}>u_{2}>\cdots$. If $u_{1} \in \operatorname{Irr}(\mathrm{S})$, then let $f_{1}:=f-c_{1} u_{1}$. If $u_{1} \notin \operatorname{Irr}(S)$, that is, there exists $s_{1} \in S$ such that $u_{1}=\left.q_{1}\right|_{s_{1}}$ and $\left.q_{1}\right|_{s_{1}}$ is normal, then let $f_{1}:=f-\left.c_{1} q_{1}\right|_{s_{1}}$. In both cases $\overline{f_{1}}<\bar{f}$. By the minimality of $f$, we have that $f_{1}$ has the desired expression. Then $f$ also has the desired expression. This is a contradiction.

Now we are ready to derive the Composition-Diamond Lemma.

Theorem 4.8. (Composition-Diamond Lemma) Let $\geq$ be the weakly monomial order on $\mathcal{B}\left(\Delta_{n} X\right)$ defined in Eq. (26), $S_{n}$ a set of monic DRB polynomials in $\mathbf{k} \mathcal{B}\left(\Delta_{n} X\right)$ and $\operatorname{Id}\left(\mathrm{S}_{\mathrm{n}}\right)$ the Rota-Baxter ideal of $\mathbf{k} \mathcal{B}\left(\Delta_{n} X\right)$ generated by $S_{n}$. Then the following conditions are equivalent:

(a) $S_{n}$ is a Gröbner-Shirshov basis in $\mathbf{k} \mathcal{B}\left(\Delta_{n} X\right)$.

(b) If $0 \neq f \in \operatorname{Id}\left(\mathrm{S}_{\mathrm{n}}\right)$, then $\bar{f}=\left.q\right|_{s}$ for some $q \in \mathcal{B}^{\star}\left(\Delta_{n} X\right)$, $s \in S_{n}$ and $\left.q\right|_{s}$ is normal.

(c) $\operatorname{Irr}\left(\mathrm{S}_{\mathrm{n}}\right):=\mathcal{B}\left(\Delta_{n} X\right) \backslash\left\{\left.q\right|_{s}\left|q \in \mathcal{B}^{\star}\left(\Delta_{n} X\right), s \in S_{n}, q\right|_{s}\right.$ is normal $\}$ is a $\mathbf{k}$-basis of $\mathbf{k} \mathcal{B}\left(\Delta_{n} X\right) / \operatorname{Id}\left(\mathrm{S}_{\mathrm{n}}\right)$. In other words, $\mathbf{k} \operatorname{Irr}\left(\mathrm{S}_{\mathrm{n}}\right) \oplus \operatorname{Id}\left(\mathrm{S}_{\mathrm{n}}\right)=\mathbf{k} \mathcal{B}\left(\Delta_{n} X\right)$.

Proof. (a) $\Rightarrow$ (b): Let $0 \neq f \in \operatorname{Id}\left(\mathrm{S}_{\mathrm{n}}\right)$. Then by Lemmas 3.3 and 4.5,

$$
f=\left.\sum_{i=1}^{k} c_{i} q_{i}\right|_{s_{i}}, \text { where } 0 \neq c_{i} \in \mathbf{k}, s_{i} \in S_{n},\left.q_{i}\right|_{s_{i}} \text { is normal, } 1 \leq i \leq k .
$$

Let $w_{i}=\left.q_{i}\right|_{i}, 1 \leq i \leq k$. We rearrange them in non-increasing order by

$$
w_{1}=w_{2}=\cdots=w_{m}>w_{m+1} \geq \cdots \geq w_{k} .
$$

If for each $0 \neq f \in \operatorname{Id}\left(\mathrm{S}_{\mathrm{n}}\right)$, there is a choice of the above sum such that $m=1$, then $\bar{f}=\left.q_{1}\right|_{s_{1}}$ and we are done. So suppose the implication (a) $\Rightarrow(b)$ does not hold. Then there is a $0 \neq f \in \operatorname{Id}\left(\mathrm{S}_{\mathrm{n}}\right)$ such that for any expression in Eq. (27), we have that $m \geq 2$. Fix such an $f$ and choose an expression in Eq. (27) such that $\left.q_{1}\right|_{\overline{s_{1}}}$ is minimal and then with $m \geq 2$ minimal, that is, with the fewest $\left.q_{i}\right|_{s_{i}}$ such that $\left.q_{i}\right|_{\bar{s}_{i}}=\left.q_{1}\right|_{\overline{s_{1}}}$. Since $m \geq 2$, we have $\left.q_{1}\right|_{\overline{s_{1}}}=w_{1}=w_{2}=\left.q_{2}\right|_{\overline{s_{2}}}$.

Since $S_{n}$ is a Gröbner-Shirshov basis in $\mathbf{k} \mathcal{B}\left(\Delta_{n} X\right)$, by Lemma 4.6, we have

$$
\left.q_{2}\right|_{\overline{s_{2}}}-\left.q_{1}\right|_{\overline{s_{1}}}=\left.\sum_{j} d_{j} p_{j}\right|_{r_{j}}
$$

where $d_{j} \in \mathbf{k}, r_{j} \in S_{n}, p_{j} \in \mathcal{B}^{\star}(\Delta X)$ and $\left.p_{j}\right|_{r_{j}}$ are normal with $\left.p_{j}\right|_{r_{j}}<w_{1}$. Hence

$$
f=\left.\sum_{i=1}^{k} c_{i} q_{i}\right|_{s_{i}}=\left.\left(c_{1}+c_{2}\right) q_{1}\right|_{s_{1}}+\left.c_{3} q_{3}\right|_{s_{3}}+\cdots+\left.c_{m} q_{m}\right|_{s_{m}}+\left.\sum_{i=m+1}^{k} c_{i} q_{i}\right|_{s_{i}}+\left.\sum_{j} c_{2} d_{j} p_{j}\right|_{r_{j}} .
$$

By the minimality of $m$, we must have $c_{1}+c_{2}=c_{3}=\cdots=c_{m}=0$. Then we obtain an expression of $f$ in the form of Eq. (27) for which $\left.q_{1}\right|_{s_{1}}$ is even smaller, a contradiction. 
(b) $\Rightarrow(c)$ : Obviously $0 \in \mathbf{k} \operatorname{Irr}\left(\mathrm{S}_{\mathrm{n}}\right)+\operatorname{Id}\left(\mathrm{S}_{\mathrm{n}}\right) \subseteq \mathbf{k} \mathcal{B}\left(\Delta_{n} X\right)$. Suppose the inclusion is proper. Then $\mathbf{k} \mathcal{B}\left(\Delta_{n} X\right) \backslash\left(\mathbf{k} \operatorname{Irr}\left(\mathrm{S}_{\mathrm{n}}\right)+\operatorname{Id}\left(\mathrm{S}_{\mathrm{n}}\right)\right)$ contains only nonzero elements. Let $f \in \mathbf{k} \mathcal{B}\left(\Delta_{n} X\right) \backslash\left(\mathbf{k} \operatorname{Irr}\left(\mathrm{S}_{\mathrm{n}}\right)+\operatorname{Id}\left(\mathrm{S}_{\mathrm{n}}\right)\right)$ be such that

$$
\bar{f}=\min \left\{\bar{g} \mid g \in \mathbf{k} \mathcal{B}\left(\Delta_{n} X\right) \backslash\left(\operatorname{kirr}\left(\mathrm{S}_{\mathrm{n}}\right)+\operatorname{Id}\left(\mathrm{S}_{\mathrm{n}}\right)\right)\right\} .
$$

Case I. $\bar{f} \in \operatorname{Irr}\left(\mathrm{S}_{\mathrm{n}}\right)$. Then $f \neq \bar{f}$ since $f \notin \operatorname{Irr}\left(\mathrm{S}_{\mathrm{n}}\right)$. By $\overline{f-\bar{f}}<\bar{f}$ and the minimality of $\bar{f}$, we must have $f-\bar{f} \in \mathbf{k} \operatorname{Irr}\left(\mathrm{S}_{\mathrm{n}}\right)+\operatorname{Id}\left(\mathrm{S}_{\mathrm{n}}\right)$ and so $f \in \mathbf{k} \operatorname{Irr}\left(\mathrm{S}_{\mathrm{n}}\right)+\operatorname{Id}\left(\mathrm{S}_{\mathrm{n}}\right)$, a contradiction.

Case II. $\bar{f} \notin \operatorname{Irr}\left(\mathrm{S}_{\mathrm{n}}\right)$. Then by the definition of $\operatorname{Irr}\left(\mathrm{S}_{\mathrm{n}}\right)$, we have $\bar{f}=\left.q\right|_{\bar{s}}$ for some $q \in \mathcal{B}^{\star}(\Delta X)$, $s \in S_{n}$ and $\left.q\right|_{s}$ is normal. Thus $\overline{\left.q\right|_{s}}=\left.q\right|_{s}=\bar{f}$ and so $\overline{f-\left.q\right|_{s}}<\bar{f}$. If $f=\left.q\right|_{s}$, then $f \in \operatorname{Id}\left(\mathrm{S}_{\mathrm{n}}\right)$, a contradiction. If $f \neq\left. q\right|_{s}$, then $f-\left.q\right|_{s} \neq 0$ with $\overline{f-\left.q\right|_{s}}<\bar{f}$. By the minimality of $\bar{f}$, we have $f-\left.q\right|_{s} \in \operatorname{kIrr}\left(\mathrm{S}_{\mathrm{n}}\right)+\operatorname{Id}\left(\mathrm{S}_{\mathrm{n}}\right)$. This implies that $f \in \mathbf{k} \operatorname{Irr}\left(\mathrm{S}_{\mathrm{n}}\right)+\operatorname{Id}\left(\mathrm{S}_{\mathrm{n}}\right)$, again a contradiction.

Hence $\mathbf{k} \operatorname{Irr}\left(\mathrm{S}_{\mathrm{n}}\right)+\operatorname{Id}\left(\mathrm{S}_{\mathrm{n}}\right)=\mathbf{k} \mathcal{B}\left(\Delta_{n} X\right)$. Suppose $\mathbf{k} \operatorname{Irr}\left(\mathrm{S}_{\mathrm{n}}\right) \cap \operatorname{Id}\left(\mathrm{S}_{\mathrm{n}}\right) \neq 0$ and let $0 \neq f \in \mathbf{k} \operatorname{Irr}\left(\mathrm{S}_{\mathrm{n}}\right) \cap$ $\operatorname{Id}\left(\mathrm{S}_{\mathrm{n}}\right)$. Then

$$
f=c_{1} v_{1}+c_{2} v_{2}+\cdots+c_{k} v_{k},
$$

where $v_{1}>v_{2}>\cdots>v_{k} \in \operatorname{Irr}\left(\mathrm{S}_{\mathrm{n}}\right)$. Since $f \in \operatorname{Id}\left(\mathrm{S}_{\mathrm{n}}\right)$, by Item (b), we have $v_{1}=\bar{f}=\left.q\right|_{s}$ for some $q \in \mathcal{B}^{\star}\left(\Delta_{n} X\right), s \in S_{n}$ and $\left.q\right|_{s}$ is normal. This is a contradiction to the construction of $\operatorname{Irr}\left(\mathrm{S}_{\mathrm{n}}\right)$. Therefore $\mathbf{k} \operatorname{Irr}\left(\mathrm{S}_{\mathrm{n}}\right) \oplus \operatorname{Id}\left(\mathrm{S}_{\mathrm{n}}\right)=\mathbf{k} \mathcal{B}\left(\Delta_{n} X\right)$ and $\operatorname{Irr}\left(\mathrm{S}_{\mathrm{n}}\right)$ is a $\mathbf{k}$-basis of $\mathbf{k} \mathcal{B}(\Delta X) / \operatorname{Id}\left(\mathrm{S}_{\mathrm{n}}\right)$.

(c) $\Rightarrow(a)$ : Suppose $f, g \in S_{n}$ give an intersection or including composition. Let $F=f u$ and $G=v g$ in the case of intersection composition and let $F=f$ and $G=\left.q\right|_{g}$ in the case of including composition. Then we have $w:=\bar{F}=\bar{G}$. If $(f, g)_{w}=F-G=0$, then there is nothing to prove. If $(f, g)_{w} \neq 0$, then we have

$$
(f, g)_{w}=\sum_{i} c_{i} q_{i}, \quad 0 \neq c_{i} \in \mathbf{k}, q_{1}>q_{2}>\cdots>q_{k} \in \mathcal{B}\left(\Delta_{n} X\right) .
$$

Then $q_{i}<\bar{F}=\bar{G}=w$. Since $(f, g)_{w} \in \operatorname{Id}\left(\mathrm{S}_{\mathrm{n}}\right)$, by Item(c), we have that the $q_{i}$ are not in $\operatorname{Irr}\left(\mathrm{S}_{\mathrm{n}}\right)$. By the definition of $\operatorname{Irr}\left(\mathrm{S}_{\mathrm{n}}\right)$, there are $q_{i} \in \mathcal{B}^{\star}\left(\Delta_{n} X\right), s_{i} \in S_{n}$ such that $q_{i}=\left.q_{i}\right|_{s_{i}}$ and $\left.q_{i}\right|_{s_{i}}$ is normal. Since $\overline{\left.q_{i}\right|_{s_{i}}}=\left.q_{i}\right|_{\overline{S_{i}}}<w$, we have $(f, g)_{w} \equiv 0 \bmod \left(S_{n}, w\right)$.

For any composition of multiplication $f u$ where $f \in S_{n}$ and $u \in C\left(\Delta_{n} X\right) \mathcal{B}\left(\Delta_{n} X\right)$, we have $f u \in$ $\operatorname{Id}\left(S_{n}\right)$. By Lemma 4.7, it follows that $f u=\left.\sum_{i} c_{i} q_{i}\right|_{s_{i}}$ where $0 \neq c_{i} \in \mathbf{k}, s_{i} \in S_{n}, q_{i} \in \mathcal{B}^{\star}\left(\Delta_{n} X\right)$, $\left.q_{i}\right|_{s_{i}}$ is normal and $\left.q_{i}\right|_{\overline{s_{i}}} \leq \overline{f u}$. Hence the composition of multiplication is trivial mod $\left[S_{n}\right]$.

For any composition of derivation $d^{\ell}(f)$ where $f \in S_{n}$ and $\ell \in \mathbb{Z}_{\geq 1}$, we have $d^{\ell}(f) \in \operatorname{Id}\left(\mathrm{S}_{\mathrm{n}}\right)$. By Lemma 4.7, we have $d^{\ell}(f)=\left.\sum_{i} c_{i} q_{i}\right|_{s_{i}}$ where $0 \neq c_{i} \in \mathbf{k}, s_{i} \in S_{n}, q_{i} \in \mathcal{B}^{\star}\left(\Delta_{n} X\right),\left.q_{i}\right|_{s_{i}}$ is normal and $\left.q_{i}\right|_{\overline{s_{i}}} \leq \overline{f P(v)}$. Hence the composition of derivation $d^{\ell}(f)$ is trivial $\bmod \left[S_{n}\right]$.

Therefore $S_{n}$ is a Gröbner-Shirshov basis.

\section{GRoÖBNER-SHIRSHOV BASES AND FREE COMMUTATIVE INTEGRO-DIFFERENTIAL ALGEBRAS}

In this section we begin with a finite set $X$ and prove that the relation ideal of the free commutative differential Rota-Baxter algebra on $X$ of order $n$, where $n \geq 1$, that defines the corresponding commutative integro-differential algebra of order $n$ possesses a Gröbner-Shirshov basis. This is done in Section 5.1. Then in Section 5.2, we apply the Composition-Diamond Lemma in Theorem 4.8 to construct a canonical basis for the commutative integro-differential algebra of order $n$. Taking $n$ to go to the infinity, we obtain a canonical basis of the free commutative integrodifferential algebra on the finite set $X$. Finally for any well-ordered set $X$, by showing that the canonical basis of the free commutative integro-differential algebra on each finite subset of $X$ is 
compatible with the inclusion of the subset in $X$, we obtain a canonical basis of the free commutative integro-differential algebra on $X$.

5.1. Gröbner-Shirshov basis. We begin with a lemma that simplifies the defining ideal of the integro-differential algebra.

Lemma 5.1. Let $X$ be a finite set and let $\amalg\left(\mathbf{k}\left[\Delta_{n} X\right]\right)$ be the free commutative differential RotaBaxter algebra on $X$. The differential Rota-Baxter ideal of $\amalg\left(\mathbf{k}\left[\Delta_{n} X\right]\right)$ generated by the set

$$
\left\{P(d(u) P(v))-u P(v)+P(u v)+\lambda P(d(u) v) \mid u, v \in \amalg\left(\mathbf{k}\left[\Delta_{n} X\right]\right) \cdot\right\}
$$

is generated by

(28) $S_{n}:=\left\{P(d(u) P(v))-u P(v)+P(u v)+\lambda P(d(u) v) \mid u, v \in \amalg\left(\mathbf{k}\left[\Delta_{n} X\right]\right), u \notin P\left(\amalg\left(\mathbf{k}\left[\Delta_{n} X\right]\right)\right)\right\}$.

Proof. If $u$ is in $P\left(\amalg\left(\mathbf{k}\left[\Delta_{n} X\right]\right)\right)$, let $u=P(\hat{u})$ for some $\hat{u} \in \amalg\left(\mathbf{k}\left[\Delta_{n} X\right]\right)$. Then $P(d(u) P(v))-u P(v)+$ $P(u v)+\lambda P(d(u) v)$ vanishes since $P$ is a Rota-Baxter algebra. This proves the lemma.

We show that $S_{n}$ is a Gröbner-Shirshov basis of the ideal $\operatorname{Id}\left(S_{n}\right) \subseteq \amalg\left(\mathbf{k}\left[\Delta_{n} X\right]\right)$.

Lemma 5.2. Let $\phi(u, v) \in S_{n}$ with $u \in \mathcal{B}\left(\Delta_{n} X\right) \backslash P\left(\mathcal{B}\left(\Delta_{n} X\right)\right)$ and $v \in \mathcal{B}\left(\Delta_{n} X\right)$. Then $\overline{\phi(u, v)}=$ $1 \otimes \overline{d\left(u_{0}\right)} \otimes w$ for some $u_{0} \in C\left(\Delta_{n} X\right)$ and $w \in \mathcal{B}\left(\Delta_{n} X\right)$.

Proof. Let $u=u_{0} \otimes \hat{u}$ with $1 \neq u_{0} \in C\left(\Delta_{n} X\right)$ and $\hat{u} \in \mathcal{B}\left(\Delta_{n} X\right)$ (take $\hat{u}=1 \in \mathbf{k}$ when $u \in C\left(\Delta_{n} X\right)$ ). Then

$$
\begin{aligned}
\overline{\phi(u, v)} & =\overline{P(d(u) P(v))}=\overline{P\left(d\left(u_{0} \otimes \hat{u}\right)(1 \otimes v)\right)}=\overline{P\left(d\left(u_{0}\right) \otimes\left(\hat{u} \amalg_{\lambda} v\right)\right)} \\
& =\overline{P\left(d\left(u_{0}\right) \otimes w\right)}=P\left(\overline{d\left(u_{0}\right)} \otimes w\right)=1 \otimes \overline{d\left(u_{0}\right)} \otimes w,
\end{aligned}
$$

where $w=\overline{\hat{u} \amalg_{\lambda} v} \in \mathcal{B}\left(\Delta_{n} X\right)$.

By the above lemma, we see that $\overline{\phi(u, v)} \in P\left(\mathcal{B}\left(\Delta_{n} X\right)\right)$ and so $\overline{\phi(u, v)} \notin C\left(\Delta_{n} X\right)$. So from Definition 4.1, there is no intersection compositions in $S_{n}$. The following two lemmas show that other kinds of compositions in $S_{n}$ are trivial.

Lemma 5.3. The compositions of multiplication and derivation are trivial $\bmod \left[S_{n}\right]$.

Proof. Let

$$
f:=\phi(u, v):=P(d(u) P(v))-u P(v)+P(u v)+\lambda P(d(u) v) \in S_{n},
$$

where $u \in \mathcal{B}\left(\Delta_{n} X\right) \backslash P\left(\mathcal{B}\left(\Delta_{n} X\right)\right)$ and $v \in \mathcal{B}\left(\Delta_{n} X\right)$. First, we check that the compositions of derivation are trivial $\bmod \left(S_{n}\right)$. By Eq. (6) and Eq. (8), we have

$d(f)=d(u) P(v)-d(u P(v))+u v+\lambda d(u) v=d(u) P(v)-d(u) P(v)-u v-\lambda d(u) v+u v+\lambda d(u) v=0$.

Hence $d^{\ell}(f) \equiv 0 \bmod \left[S_{n}\right]$ for any $\ell \in \geq 1$.

Next, we check that the compositions of multiplication $\phi(u, v) w_{0} P(w)$ with $w_{0} \in C\left(\Delta_{n} X\right)$ and $w \in \mathcal{B}\left(\Delta_{n} X\right)$ are trivial. Since $w_{0} \in C\left(\Delta_{n} X\right)$, it is sufficient to show that $\phi(u, v) P(w)$ is trivial. Note that $\overline{\phi(u, v)} \in P\left(\mathcal{B}\left(\Delta_{n} X\right)\right)$ by Lemma 5.2. From Eq. (7) we obtain

$$
\begin{aligned}
\phi(u, v) P(w)= & P(d(u) P(v)) P(w)-(u P(v)) P(w)+P(u v) P(w)+\lambda P(d(u) v) P(w) \\
= & P(P(d(u) P(v)) w)+P(d(u) P(v) P(w))+\lambda P(d(u) P(v) w) \\
& -u P(v) P(w)+P(u v) P(w)+\lambda P(d(u) v) P(w) \\
= & P(P(d(u) P(v)) w)+P(d(u) P(P(v) w+v P(w)+\lambda v w))+\lambda P(d(u) P(v) w) \\
& -u P(v) P(w)+P(u v) P(w)+\lambda P(d(u) v) P(w)
\end{aligned}
$$


Since $\phi(u, v)=P(d(u) P(v))-u P(v)+P(u v)+\lambda P(d(u) v)$, we have

$$
\begin{aligned}
& P(P(d(u) P(v)) w)=P(\phi(u, v) w)+P(u P(v) w)-P(P(u v) w)-\lambda P(P(d(u) v) w), \\
& P(d(u) P(P(v) w+v P(w)+\lambda v w)) \\
= & \phi(u, P(v) w+v P(w)+\lambda v w)+u P(P(v) w+v P(w)+\lambda v w) \\
& -P(u(P(v) w+v P(w)+\lambda v w))-\lambda P(d(u)(P(v) w+v P(w)+\lambda v w)) \\
= & \phi(u, P(v) w+v P(w)+\lambda v w)+u P(w P(v))+u P(v P(w))+\lambda u P(v w)-P(u w P(v)) \\
& -P(u v P(w))-\lambda P(u v w)-\lambda P(d(u) w P(v))-\lambda P(d(u) v P(w))-\lambda^{2} P(d(u) v w)
\end{aligned}
$$

and

$$
\begin{aligned}
& -u P(v) P(w)+P(u v) P(w)+\lambda P(d(u) v) P(w) \\
= & -u P(P(v) w)-u P(v P(w))-\lambda u P(v w)+P(P(u v) w)+P(u v P(w))+\lambda P(u v w) \\
& +\lambda P(P(d(u) v) w)+\lambda P(d(u) v P(w))+\lambda^{2} P(d(u) v w) .
\end{aligned}
$$

Substituting Eq. (31), Eq. (32) and Eq. (33) into Eq. (30), we have

$$
\phi(u, v) P(w)=P(\phi(u, v) w)+\phi(u, w P(v))+\phi(u, v P(w))+\lambda \phi(u, v w)
$$

The last three terms are already in $S_{n}$ and hence are of the form $\left.q\right|_{s}$ with $q=\star$ and $s \in S_{n}$. So we just need to bound the leading terms. Note that

$$
\overline{P(a P(b))}, \overline{P(b P(a))}, \overline{P(a b)} \leq \overline{P(a) P(b)} \text { for } a, b \in \mathcal{B}\left(\Delta_{n} X\right) .
$$

So we have

$$
\overline{\phi(u, w P(v))}=\overline{P(d(u) P(w P(v)))} \leq \overline{P(d(u) P(v) P(w))} \leq \overline{P(d(u) P(v)) P(w)}=\overline{\phi(u, v) P(w)} .
$$

We similarly show that $\overline{\phi(u, v P(w))}, \overline{\phi(u, v w)} \leq \overline{\phi(u, v) P(w)}$. So $\phi(u, w P(v))+\phi(u, v P(w))+$ $\lambda \phi(u, v w) \equiv 0 \bmod \left[S_{n}\right]$. Hence $\phi(u, v) P(w) \equiv 0 \bmod \left[S_{n}\right]$ if and only if $P(\phi(u, v) w) \equiv 0 \bmod$ $\left[S_{n}\right]$. We prove the latter statement by induction on $\operatorname{dep}(w)$.

If $\operatorname{dep}(w)=1$, that is, $w \in C\left(\Delta_{n} X\right)$, let $q:=P(\star w) \in \mathcal{B}^{\star}\left(\Delta_{n} X\right)$. Then $\left.q\right|_{\phi(u, v)}=P(\phi(u, v) w)$ and $\left.q\right|_{\phi(u, v)}$ is normal by $w \in C\left(\Delta_{n} X\right)$. Since

$$
\overline{P(\phi(u, v) w)}=\overline{P(\overline{\phi(u, v)} w)}=\overline{P(P(d(u) P(v)) w)} \leq \overline{P(d(u) P(v)) P(w)}=\overline{\overline{\phi(u, v)} P(w)}=\overline{\phi(u, v) P(w)},
$$

we have $P(\phi(u, v) w) \equiv 0 \bmod \left[S_{n}\right]$.

Suppose $w \in C\left(\Delta_{n} X\right) P\left(\mathcal{B}\left(\Delta_{n} X\right)\right)$ and let $w=w_{1} P(\tilde{w})$ with $w_{1} \in C\left(\Delta_{n} X\right)$ and $\tilde{w} \in \mathcal{B}\left(\Delta_{n} X\right)$. Since $\operatorname{dep}(\tilde{w})<\operatorname{dep}(w)$, by the induction hypothesis, we may assume that

$$
\phi(u, v) P(\tilde{w})=\left.\sum_{i} c_{i} p_{i}\right|_{s_{i}}
$$

where $0 \neq c_{i} \in \mathbf{k}, p_{i} \in \mathcal{B}^{\star}\left(\Delta_{n} X\right), s_{i} \in S_{n},\left.p_{i}\right|_{s_{i}}$ is normal and $\overline{\left.p_{i}\right|_{s_{i}}} \leq \overline{\phi(u, v) P(\tilde{w})}$. Let $q_{i}:=P\left(w_{1} p_{i}\right)$. Since $\left.p_{i}\right|_{s_{i}}$ is normal and $w_{1} \in C\left(\Delta_{n} X\right)$, it follows that $\left.q_{i}\right|_{s_{i}}$ is normal. Furthermore, we have

$$
P(\phi(u, v) w)=P\left(\phi(u, v) w_{1} P(\tilde{w})\right)=\sum_{i} c_{i} P\left(\left.w_{1} p_{i}\right|_{s_{i}}\right)=\left.\sum_{i} c_{i} q_{i}\right|_{s_{i}}
$$

and

$$
\overline{\left.q_{i}\right|_{s_{i}}}=\overline{P\left(\left.w_{1} p_{i}\right|_{s_{i}}\right)} \leq \overline{P\left(w_{1} \phi(u, v) P(\tilde{w})\right)}=\overline{P(\phi(u, v) w)} \leq \overline{\phi(u, v) P(w)} .
$$

Therefore $P(\phi(u, v) w) \equiv 0 \bmod \left[S_{n}\right]$. This completes the induction. Hence $\phi(u, v) P(w) \equiv 0 \bmod$ $\left[S_{n}\right]$, as needed. 
Lemma 5.4. The including compositions in $S_{n}$ are trivial.

Proof. We need to show that the ambiguities of all possible including compositions of the polynomials in $S_{n}$ are trivial. The ambiguities of all such compositions are of the form

$$
P\left(d(u) P\left(\left.q\right|_{P(d(v) P(w))}\right)\right) \text { and } P\left(d\left(\left.q\right|_{P(d(u) P(v))}\right) P(w)\right) \text {. }
$$

Let two elements $f$ and $g$ of $S_{n}$ be given. They are of the form

$$
f:=\phi(u, v), \quad g:=\phi(r, s), \quad u, v \in \mathcal{B}\left(\Delta_{n} X\right) \backslash P\left(\mathcal{B}\left(\Delta_{n} X\right)\right) \text { and } r, s \in \mathcal{B}\left(\Delta_{n} X\right) .
$$

Case I. Suppose $v=\left.p\right|_{\bar{g}}=\left.p\right|_{\overline{\phi(r, s)}}=\left.p\right|_{P(d(r) P(s))}$ for some $p \in \mathcal{B}^{\star}\left(\Delta_{n} X\right)$ and

$$
w:=\bar{f}=\overline{\phi(u, v)}=\overline{P(d(u) P(v))}=\overline{P(d(u) P(p \mid \bar{g}))}=\overline{\left.q\right|_{\bar{g}}}=\left.q\right|_{\bar{g}},
$$

with $q=P(d(u) P(p)) \in \mathcal{B}^{\star}\left(\Delta_{n} X\right)$ and $\left.q\right|_{g}$ being normal. Then

$$
f=\phi(u, v)=P\left(d(u) P\left(\left.p\right|_{P(d(r) P(s))}\right)\right)-u P\left(\left.p\right|_{P(d(r) P(s))}\right)+P\left(\left.u p\right|_{P(d(r) P(s))}\right)+\lambda P\left(\left.d(u) p\right|_{P(d(r) P(s))}\right)
$$

and

$$
\left.q\right|_{g}=\left.q\right|_{\phi(r, s)}=P\left(d(u) P\left(\left.p\right|_{P(d(r) P(s))}\right)\right)-P\left(d(u) P\left(\left.p\right|_{r P(s)}\right)\right)+P\left(d(u) P\left(\left.p\right|_{P(r s)}\right)\right)+\lambda P\left(d(u) P\left(\left.p\right|_{P(d(r) s)}\right)\right) .
$$

So we have

$$
\begin{aligned}
(f, g)_{w}:= & f-\left.q\right|_{g} \\
= & -u P\left(\left.p\right|_{P(d(r) P(s))}\right)+P\left(\left.u p\right|_{P(d(r) P(s))}\right)+\lambda P\left(\left.d(u) p\right|_{P(d(r) P(s))}\right) \\
& +P\left(d(u) P\left(\left.p\right|_{r P(s)}\right)\right)-P\left(d(u) P\left(\left.p\right|_{P(r s)}\right)\right)-\lambda P\left(d(u) P\left(\left.p\right|_{P(d(r) s)}\right)\right) .
\end{aligned}
$$

Since $\phi(u, v)=P(d(u) P(v))-u P(v)+P(u v)+\lambda P(d(u) v)$, we have

$$
\begin{aligned}
-u P\left(\left.p\right|_{P(d(r) P(s))}\right) & =-u P\left(\left.p\right|_{\phi(r, s)}\right)-u P\left(\left.p\right|_{r P(s)}\right)+u P\left(\left.p\right|_{P(r s)}\right)+\lambda u P\left(\left.p\right|_{P(d(r) s)}\right) \\
P\left(\left.u p\right|_{P(d(r) P(s))}\right) & =+P\left(\left.u p\right|_{\phi(r, s)}\right)+P\left(\left.u p\right|_{r P(s)}\right)-P\left(\left.u p\right|_{P(r s)}\right)-\lambda P\left(\left.u p\right|_{P(d(r) s)}\right) \\
\lambda P\left(\left.d(u) p\right|_{P(d(r) P(s))}\right) & =+\lambda P\left(\left.d(u) p\right|_{\phi(r, s)}\right)+\lambda P\left(\left.d(u) p\right|_{r P(s)}\right)-\lambda P\left(\left.d(u) p\right|_{P(r s)}\right)-\lambda^{2} P\left(\left.d(u) p\right|_{P(d(r) s)}\right) \\
P\left(d(u) P\left(\left.p\right|_{r(s)}\right)\right) & =\phi\left(u,\left.p\right|_{r(s)}\right)+u P\left(\left.p\right|_{r P(s)}\right)-P\left(\left.u p\right|_{r(s)}\right)-\lambda P\left(\left.d(u) p\right|_{r P(s)}\right) \\
-P\left(d(u) P\left(\left.p\right|_{P(r s)}\right)\right) & =-\phi\left(u,\left.p\right|_{P(r s)}\right)-u P\left(\left.p\right|_{P(r s)}\right)+P\left(\left.u p\right|_{P(r s)}\right)+\lambda P\left(\left.d(u) p\right|_{P(r s)}\right) \\
-\lambda P\left(d(u) P\left(\left.p\right|_{P(d(r) s)}\right)\right) & =-\lambda \phi\left(u,\left.p\right|_{P(d(r) s)}\right)-\lambda u P\left(\left.p\right|_{P(d(r) s)}\right)+\lambda P\left(\left.u p\right|_{P(d(r) s)}\right)+\lambda^{2} P\left(\left.d(u) p\right|_{P(d(r) s)}\right) .
\end{aligned}
$$

From Eq. (34) and Eq. (35), it follows that

$(f, g)_{w}=-u P\left(\left.p\right|_{\phi(r, s)}\right)+P\left(\left.u p\right|_{\phi(r, s)}\right)+\lambda P\left(\left.d(u) p\right|_{\phi(r, s)}\right)+\phi\left(u,\left.p\right|_{r P(s)}\right)-\phi\left(u,\left.p\right|_{P(r s)}\right)-\lambda \phi\left(u,\left.p\right|_{P(d(r) s)}\right)$.

By Lemma 3.3, we have

$$
u P\left(\left.p\right|_{\phi(r, s)}\right), P\left(\left.u p\right|_{\phi(r, s)}\right), \lambda P\left(\left.d(u) p\right|_{\phi(r, s)}\right) \in I d\left(S_{n}\right)
$$

and

Since

$$
\phi\left(u,\left.p\right|_{r P(s)}\right), \phi\left(u,\left.p\right|_{P(r s)}\right), \phi\left(u,\left.p\right|_{P(d(r) s)}\right) \in S_{n} \subseteq I d\left(S_{n}\right) .
$$

$$
\overline{u P\left(\left.p\right|_{\phi(r, s)}\right)}, \quad \overline{P\left(\left.u p\right|_{\phi(r, s)}\right)}, \quad \overline{P\left(\left.d(u) p\right|_{\phi(r, s)}\right)}<\overline{\phi\left(u,\left.p\right|_{\phi(r, s)}\right)}=\overline{\phi(u, v)}=w
$$

and

$$
\overline{\phi\left(u,\left.p\right|_{r P(s)}\right)}, \quad \overline{\phi\left(u,\left.p\right|_{P(r s)}\right)}, \quad \overline{\phi\left(u,\left.p\right|_{P(d(r) s)}\right)}<\overline{\phi\left(u,\left.p\right|_{\overline{\phi(r, s)}}\right.}=\overline{\phi(u, v)}=w,
$$

we have that $(f, g)_{w} \equiv 0 \bmod \left(S_{n}, w\right)$.

Case II. Suppose $u=\left.p\right|_{\bar{g}}=\left.p\right|_{\overline{\phi(r, s)}}=\left.p\right|_{P(d(r) P(s))}$ for some $p \in \mathcal{B}^{\star}\left(\Delta_{n} X\right)$ and

$$
w:=\bar{f}=\overline{\phi(u, v)}=\overline{P(d(u) P(v))}=\overline{P\left(d\left(\left.p\right|_{\overline{\phi(r, s)}}\right) P(v)\right)}=\overline{\left.q\right|_{\bar{g}}}=\left.q\right|_{\bar{g}},
$$


with $q=P(d(p) P(v)) \in \mathcal{B}^{\star}\left(\Delta_{n} X\right)$ and $\left.q\right|_{g}$ being normal. Then

$$
f=\phi(u, v)=P\left(d\left(\left.p\right|_{P(d(r) P(s))}\right) P(v)\right)-\left.p\right|_{P(d(r) P(s))} P(v)+P\left(\left.p\right|_{P(d(r) P(s))} v\right)+\lambda P\left(d\left(\left.p\right|_{P(d(r) P(s))}\right) v\right)
$$

and

$$
\left.q\right|_{g}=\left.q\right|_{\phi(r, s)}=P\left(d\left(\left.p\right|_{P(d(r) P(s))}\right) P(v)\right)-P\left(d\left(\left.p\right|_{r P(s)}\right) P(v)\right)+P\left(d\left(\left.p\right|_{P(r s)}\right) P(v)\right)+\lambda P\left(d\left(\left.p\right|_{P(d(r) s)}\right) P(v)\right) .
$$

We have

$$
\begin{aligned}
(f, g)_{w}:= & f-\left.q\right|_{g} \\
= & -\left.p\right|_{P(d(r) P(s))} P(v)+P\left(\left.p\right|_{P(d(r) P(s))} v\right)+\lambda P\left(d\left(\left.p\right|_{P(d(r) P(s))}\right) v\right) \\
& +P\left(d\left(\left.p\right|_{r P(s)}\right) P(v)\right)-P\left(d\left(\left.p\right|_{P(r s)}\right) P(v)\right)-\lambda P\left(d\left(\left.p\right|_{P(d(r) s)}\right) P(v)\right) \\
= & -\left.p\right|_{\phi(r, s)} P(v)-\left.p\right|_{r P(s)} P(v)+\left.p\right|_{P(r s)} P(v)+\left.\lambda p\right|_{P(d(r) s)} P(v) \\
& +P\left(\left.p\right|_{\phi(r, s)} v\right)+P\left(\left.p\right|_{r P(s)} v\right)-P\left(\left.p\right|_{P(r s)} v\right)-\lambda P\left(\left.p\right|_{P(d(r) s)} v\right) \\
& +\lambda P\left(d\left(\left.p\right|_{\phi(r, s)}\right) v\right)+\lambda P\left(d\left(\left.p\right|_{r P(s)}\right) v\right)-\lambda P\left(d\left(\left.p\right|_{P(r s)}\right) v\right)-\lambda^{2} P\left(d\left(\left.p\right|_{P(d(r) s)}\right) v\right) \\
& +\phi\left(\left.p\right|_{r P(s)}, v\right)+\left.p\right|_{r P(s)} P(v)-P\left(\left.p\right|_{r P(s)} v\right)-\lambda P\left(d\left(\left.p\right|_{r(s)}\right) v\right) \\
& -\phi\left(\left.p\right|_{P(r s)}, v\right)-\left.p\right|_{P(r s)} P(v)+P\left(\left.p\right|_{P(r s)} v\right)+\lambda P\left(d\left(\left.p\right|_{P(r s)}\right) v\right) \\
& -\lambda \phi\left(\left.p\right|_{P(d(r) s)}, v\right)-\left.\lambda p\right|_{P(d(r) s)} P(w)+\lambda P\left(\left.p\right|_{P(d(r) s)} v\right)+\lambda^{2} P\left(d\left(\left.p\right|_{P(d(r) s)}\right) v\right) \\
= & -\left.p\right|_{\phi(r, s)} P(v)+P\left(\left.p\right|_{\phi(r, s)} v\right)+\lambda P\left(d\left(\left.p\right|_{\phi(r, s)}\right) v\right)+\phi\left(\left.p\right|_{r P(s)}, v\right)-\phi\left(\left.p\right|_{P(r s)}, v\right)-\lambda \phi\left(\left.p\right|_{P(d(r) s), v) .}\right.
\end{aligned}
$$

By Lemma 3.3, we have

$$
\left.p\right|_{\phi(r, s)} P(v), P\left(\left.p\right|_{\phi(r, s)} v\right), P\left(d\left(\left.p\right|_{\phi(r, s)}\right) v\right) \in \operatorname{Id}\left(S_{n}\right)
$$

and

$$
\phi\left(\left.p\right|_{r P(s)}, v\right), \phi\left(\left.p\right|_{P(r s)}, v\right), \phi\left(\left.p\right|_{P(d(r) s)}, v\right) \in S_{n} \subseteq I d\left(S_{n}\right)
$$

Since

$$
\overline{\left.p\right|_{\phi(r, s)} P(v)}, \quad \overline{P\left(\left.p\right|_{\phi(r, s)} v\right)}, \quad \overline{P\left(d\left(\left.p\right|_{\phi(r, s)}\right) v\right)}<\overline{\phi\left(\left.p\right|_{\phi(r, s)}, v\right)}=\overline{\phi(u, v)}=w
$$

and

$$
\overline{\phi\left(\left.p\right|_{r P(s)}, v\right)}, \quad \overline{\phi\left(\left.p\right|_{P(r s)}, v\right)}, \quad \overline{\phi\left(\left.p\right|_{P(d(r) s)}, v\right)}<\overline{\phi\left(\left.p\right|_{\overline{\phi(r, s)}}, v\right)}=\overline{\phi(u, v)}=w,
$$

it follows that $(f, g)_{w} \equiv 0 \bmod \left(S_{n}, w\right)$.

By the remark before Lemma 5.3, Lemmas 5.3 and 5.4, it follows immediately that

Theorem 5.5. $S_{n}$ is a Gröbner-Shirshov basis in $\mathbf{k} \mathcal{B}\left(\Delta_{n} X\right)$. Hence $\operatorname{Irr}\left(S_{n}\right)$ in Theorem 4.8 is a linear basis of $\amalg\left(\mathbf{k}\left[\Delta_{n} X\right]\right) / \operatorname{Id}\left(S_{n}\right)$.

5.2. Bases for free commutative integro-differential algebras. We next identify $\operatorname{Irr}\left(S_{n}\right)$ and thus obtaining a canonical basis of $\amalg\left(\mathbf{k}\left[\Delta_{n} X\right]\right) / \operatorname{Id}\left(S_{n}\right)$.

Lemma 5.6. Let $\leq$ be the linear order on $C(\Delta X)$ defined in Eqs. (25) and (19), and $u=u_{0} u_{1} \cdots u_{k} \in$ $C(\Delta X)$ with $u_{0}, \cdots, u_{k} \in \Delta X$ and $u_{0} \geq \cdots \geq u_{k}$. Then $\overline{d_{X}(u)}=u_{0} u_{1} \cdots u_{k-1} d_{X}\left(u_{k}\right)$. If $u$ is in $C\left(\Delta_{n} X\right)$, then $\overline{d_{X}(u)}=u_{0} u_{1} \cdots u_{k-1} d_{X}\left(u_{k}\right)$ provided $u_{k} \in \Delta_{n-1} X$.

Proof. We prove the first statement by induction on $k \geq 0$. If $k=0$, then $u=u_{0} \in \Delta X$ and there is nothing to prove.

Assume the result holds for $k \leq m$, where $m \geq 0$, and consider the case when $k=m+1$. Then $u=u_{0} u_{1} \cdots u_{m+1}$ with $u_{0}, \cdots, u_{m+1} \in \Delta X$ and $u_{0} \geq \cdots \geq u_{m+1}$. Let $\hat{u}=u_{0} u_{1} \cdots u_{m}$. Then

$$
d_{X}(u)=d_{X}\left(\hat{u} u_{m+1}\right)=\hat{u} d_{X}\left(u_{m+1}\right)+d_{X}(\hat{u}) u_{m+1}+\lambda d_{X}(\hat{u}) d\left(u_{m+1}\right) .
$$


By the induction hypothesis, we have $\overline{d_{X}(\hat{u})}=u_{0} u_{1} \cdots d_{X}\left(u_{m}\right)$. So $\overline{d_{X}(\hat{u}) u_{m+1}}=u_{0} u_{1} \cdots d_{X}\left(u_{m}\right) u_{m+1}$ and $\overline{d_{X}(\hat{u}) d_{X}\left(u_{m+1}\right)}=u_{0} u_{1} \cdots d_{X}\left(u_{m}\right) d_{X}\left(u_{m+1}\right)$. If $d_{X}\left(u_{m}\right) \geq u_{m+1}$, then since $u_{m}>d_{X}\left(u_{m}\right)$ and $u_{m+1}>d_{X}\left(u_{m+1}\right)$, we have

$$
\hat{u} d_{X}\left(u_{m+1}\right)=u_{0} u_{1} \cdots u_{m} d_{X}\left(u_{m+1}\right)>u_{0} u_{1} \cdots d_{X}\left(u_{m}\right) u_{m+1}>u_{0} u_{1} \cdots d_{X}\left(u_{m}\right) d_{X}\left(u_{m+1}\right)
$$

and so $\overline{d_{X}(u)}=u_{0} u_{1} \cdots u_{m} d_{X}\left(u_{m+1}\right)$. If $u_{m+1}>d_{X}\left(u_{m}\right)$ and $u_{m}>u_{m+1}$, then since $u_{m}>d_{X}\left(u_{m}\right)$, we have

$$
\hat{u} d_{X}\left(u_{m+1}\right)=u_{0} u_{1} \cdots u_{m} d_{X}\left(u_{m+1}\right)>u_{0} u_{1} \cdots u_{m+1} d_{X}\left(u_{m}\right), u_{0} u_{1} \cdots d_{X}\left(u_{m}\right) d_{X}\left(u_{m+1}\right)
$$

and hence $\overline{d_{X}(u)}=u_{0} u_{1} \cdots u_{m} d_{X}\left(u_{m+1}\right)$. If $u_{m+1}>d_{X}\left(u_{m}\right)$ and $u_{m}=u_{m+1}$, then since $u_{m}>d_{X}\left(u_{m}\right)$, we have

$$
\hat{u} d_{X}\left(u_{m+1}\right)=u_{0} u_{1} \cdots u_{m} d_{X}\left(u_{m+1}\right)=u_{0} u_{1} \cdots u_{m+1} d_{X}\left(u_{m}\right)>u_{0} u_{1} \cdots d_{X}\left(u_{m}\right) d_{X}\left(u_{m+1}\right)
$$

and so $\overline{d_{X}(u)}=u_{0} u_{1} \cdots u_{m} d_{X}\left(u_{m+1}\right)$. This completes the induction. The proof of the second statement then follows since under the condition $u_{k} \in \Delta_{n-1} X, d_{X}\left(u_{k}\right)$ does not change in $\Delta X$ or in $\Delta_{n} X$.

We now give the key concept to define $\operatorname{Irr}\left(S_{n}\right)$.

Definition 5.7. Let $u \in C(\Delta X)$ with standard form in Eq. (20):

$$
u=u_{0}^{j_{0}} \cdots u_{k}^{j_{k}} \text {, where } u_{0}, \cdots, u_{k} \in \Delta X, u_{0}>\cdots>u_{k} \text { and } j_{0}, \cdots, j_{k} \in \mathbb{Z}_{\geq 1} .
$$

Call $u$ functional if either $u \in\{1\} \cup X$ or $j_{k}>1$. Denote

$$
\mathcal{A}_{f}:=\left\{u \in C\left(\Delta_{n} X\right) \mid u \text { is functional }\right\}, \quad \mathbf{k}\{X\}_{f}:=\mathbf{k} \mathcal{A}_{f} \text { and } A_{f, 0}=\mathbf{k}\left(\mathcal{A}_{f} \backslash\{1\}\right) .
$$

Proposition 5.8. Let $X$ be a finite well-ordered set. Let $\left(A, d_{X}\right):=\left(\mathbf{k}\{X\}, d_{X}\right):=\left(\mathbf{k}[\Delta X], d_{X}\right)$ be the free commutative differential algebra on $X$. Then $A=A_{f} \oplus d_{X}(A)$.

Proof. We prove the result by induction on $|X| \geq 1$. The case when $|X|=1$ has been proved in [23]. Suppose the result holds for all $X$ such that $|X|<m$ and consider the case when $|X|=m$. Let $X=\left\{x_{1}, x_{2}, \cdots, x_{m}\right\}$ with $x_{1}>\cdots>x_{m}, B=\mathbf{k}\left\{x_{1}, \cdots, x_{m-1}\right\}$ and $C=\mathbf{k}\left\{x_{m}\right\}$. Also denote

$$
A_{f}:=\mathbf{k}\{X\}_{f}, B_{f}:=\mathbf{k}\left\{x_{1}, \cdots, x_{m-1}\right\}_{f}, C_{f}:=\mathbf{k}\left\{x_{m}\right\}_{f}, C_{f, 0}=\mathbf{k}\left\{x_{m}\right\}_{f, 0} .
$$

By the induction hypothesis, we have

$$
B=B_{f} \oplus d_{X}(B) \text { and } C=C_{f} \oplus d_{X}(C) .
$$

Then by the definition of $A_{f}$, we have

(36) $A_{f}=\left(B_{f} \otimes \mathbf{k}\right) \oplus\left(B \otimes C_{f, 0}\right)=\left(B_{f} \otimes \mathbf{k}\right) \oplus\left(B_{f} \otimes C_{f, 0}\right) \oplus\left(d_{X}(B) \otimes C_{f, 0}\right)=\left(B_{f} \otimes C_{f}\right) \oplus\left(d_{X}(B) \otimes C_{f, 0}\right)$.

Therefore $B_{f}=B_{f} \otimes 1 \subseteq A_{f}$ and $C_{f}=1 \otimes C_{f} \subseteq A_{f}$. Thus $B=B_{f} \oplus d_{X}(B) \subseteq A_{f}+d_{X}(A)$ and $C=C_{f} \oplus d_{X}(C) \subseteq A_{f}+d_{X}(A)$. Since $A=B \otimes C$ is generated as an algebra by $B \otimes 1$ and $1 \otimes C$, we have $A \subseteq A_{f}+d_{X}(A)$ and so $A_{f}+d_{X}(A)=A$.

We are left to show that $A_{f} \cap d_{X}(A)=0$. Let $\mathcal{B}:=B \cap C(\Delta X)$ (resp. $\mathcal{B}_{f}:=B_{f} \cap C(\Delta X)$, resp. $\mathcal{C}:=C \cap C(\Delta X)$, resp. $\left.\mathcal{C}_{f}:=C_{f} \cap C(\Delta X)\right)$ be the basis of monomials of $B$ (resp. $B_{f}$, resp. $C$, resp. $\left.C_{f}\right)$. Then a nonzero element $w$ of $A=B \otimes C$ is a sum

$$
w=\sum_{i=1}^{k} u_{i} \otimes \sum_{j=1}^{n_{i}} \alpha_{i j} v_{i j}=\sum_{i, j} \alpha_{i j} u_{i} \otimes v_{i j}
$$


where $u_{1}>\cdots>u_{k} \in \mathcal{B}, v_{i 1}>\cdots>v_{i n_{i}} \in \mathcal{C}, 0 \neq k_{i j} \in \mathbf{k}, 1 \leq j \leq n_{i}$. Then we have

$$
d_{X}(w)=d_{X}\left(\sum_{i, j} k_{i j} u_{i} \otimes v_{i j}\right)=\sum_{i, j} \alpha_{i j}\left(d_{X}\left(u_{i}\right) \otimes v_{i j}+u_{i} \otimes d_{X}\left(v_{i j}\right)+\lambda d_{X}\left(u_{i}\right) \otimes d_{X}\left(v_{i j}\right)\right) .
$$

We distinguish the following three cases.

Case 1. If $v_{11} \neq 1$, then the leading term in the sum in Eq. (37) is $u_{1} \otimes \overline{d_{X}\left(v_{11}\right)}$. Since $C_{f} \cap d_{X}(C)=$ 0 , we have $\overline{d_{X}\left(v_{11}\right)} \notin \mathcal{C}_{f}$. Then $u_{1} \otimes \overline{d_{X}\left(v_{11}\right)} \notin \mathcal{B} \otimes \mathcal{C}_{f}$. Since $\mathcal{B} \otimes \mathcal{C}_{f}$ is a basis of $B \otimes C_{f}$, we have $u_{1} \otimes \overline{d_{X}\left(v_{11}\right)} \notin B \otimes C_{f}$. Therefore $d_{X}\left(\sum_{i j} k_{i j} u_{i} \otimes v_{i j}\right) \notin B \otimes C_{f}$. By Eq. (36) we have

$$
B \otimes C_{f}=B_{f} \otimes C_{f} \oplus d_{X}(B) \otimes C_{f}=B_{f} \otimes C_{f} \oplus d_{X}(B) \otimes C_{f, 0} \oplus d_{X}(B) \otimes \mathbf{k}=A_{f} \oplus d_{X}(B) \otimes \mathbf{k} .
$$

Therefore $d_{X}(w) \notin A_{f}$.

Case 2. If $v_{11}=1$ and either $\overline{d_{X}\left(u_{1}\right)}>u_{2}$ or $\overline{d_{X}\left(u_{1}\right)}=u_{2}$ and $v_{21}=1$, then since $d_{X}(1)=0$, by the definition of the order defined on $\Delta X$, the leading term in the sum in Eq. (37) is $\overline{d_{X}\left(u_{1}\right)} \otimes 1$ where $\overline{d_{X}\left(u_{1}\right)} \in \mathfrak{B}$ denotes the leading term of $d_{X}\left(u_{1}\right)$. Since $B_{f} \cap d_{X}(B)=0$, we have $\overline{d_{X}\left(u_{1}\right)} \otimes 1 \notin \mathcal{B}_{f}$. Then $\overline{d_{X}\left(u_{1}\right)} \otimes 1 \notin \mathcal{B}_{f} \otimes \mathcal{C}$ and hence not in $B_{f} \otimes C$. Also $1 \notin \mathcal{C}_{f, 0}$ implies that $\overline{d_{X}\left(u_{1}\right)} \otimes 1 \notin \mathcal{B} \otimes \mathcal{C}_{f, 0}$. Here $\mathcal{C}_{f, 0}=\mathcal{C} \backslash\{1\}$ is the standard basis of $C_{f, 0}$. Thus $\overline{d_{X}\left(u_{1}\right)} \otimes 1 \notin\left(\mathcal{B}_{f} \otimes \mathcal{C}\right) \cup\left(\mathcal{B} \otimes \mathcal{C}_{f, 0}\right)$. Then we have $\overline{d_{X}\left(u_{1}\right)} \otimes 1 \notin\left(B_{f} \otimes C\right)+\left(B \otimes C_{f, 0}\right)$ and hence $d_{X}\left(\sum_{i j} k_{i j} u_{i} \otimes v_{j}\right) \notin\left(B_{f} \otimes C\right)+\left(B \otimes C_{f, 0}\right)$. Then $d_{X}(w)$ is not in $A_{f}$ by Eq. (36).

Case 3. If $v_{11}=1, \overline{d_{X}\left(u_{1}\right)}=u_{2}$ and $v_{21} \neq 1$ (note that $\overline{d_{X}\left(u_{1}\right)}<u_{2}$ is impossible since $u_{1}>u_{2}$ ), then the leading term of the sum in Eq. (37) is $u_{2} \otimes \overline{v_{21}}$. Then the proof is the same as for Case 1.

In summary, we have proved that $d_{X}(w) \notin A_{f}$ and hence $A_{f} \cap d_{X}(A)=0$.

Lemma 5.9. Let $A_{f}=\mathbf{k}\{X\}_{f}, A_{n}=\mathbf{k}\left[\Delta_{n} X\right], A_{n, f}=A_{n} \cap A_{f}$ and $d_{A_{n}}$ to be the restriction $\left.d\right|_{A_{n}}$ except $d\left(x^{(n)}\right)=0$ for $x \in X$. Then $A_{n}=A_{n, f} \oplus d_{A_{n}}\left(A_{n}\right)$.

Proof. Since $A_{n, f} \subset A_{f}, d_{A_{n}}\left(A_{n}\right) \subseteq d_{X}(A)$ and $A_{f} \cap d_{X}(A)=0$ by Proposition 5.8, we have $A_{n, f} \cap \operatorname{im}\left(d_{A_{n}}\right)=0$. Thus we only need to show $A_{n} \subseteq A_{n, f}+d_{A_{n}}\left(A_{n}\right)$ since $A_{n} \supseteq A_{n, f}+d_{A_{n}}\left(A_{n}\right)$ is clear. Suppose $A_{n} \nsubseteq A_{n, f}+d_{A_{n}}\left(A_{n}\right)$. There is a monomial $u \in C\left(\Delta_{n} X\right)$ in $A_{n} \backslash\left(A_{n, f}+d_{A_{n}}\left(A_{n}\right)\right)$ that is minimal under the order $\leq_{n}$ on $C\left(\Delta_{n} X\right)$ defined in Eqs. (19) and (25). Then $u \notin A_{f}$. Assume the minimum variable in $u$ is $x$ and $\ell$ is the highest differential order of $x$ in $u$. Then $u$ can be expressed as $u=\hat{u}\left(x^{(\ell-1)}\right)^{m} x^{(\ell)}$ with $\hat{u} \in C(\Delta X)$ and $m \geq 0$. Let $v=\hat{u}\left(x^{(\ell-1)}\right)^{m+1} \in C(\Delta X)$. By

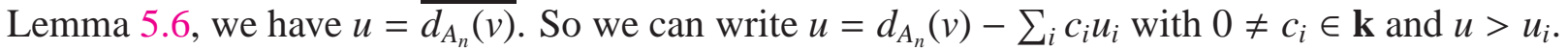
Then $d_{A_{n}}(v) \in d_{A_{n}}\left(A_{n}\right)$ and $u_{i} \in A_{n, f}+\operatorname{im}\left(d_{A_{n}}\right)$ by the minimality of $u$ in $A_{n} \backslash\left(A_{n, f}+\operatorname{im}\left(d_{A_{n}}\right)\right)$. Thus $u \in A_{n, f}+\operatorname{im}\left(d_{A_{n}}\right)$. This is a contradiction.

Lemma 5.10. (a) Let $\mathcal{A}_{d}:=\left\{\overline{d_{X}(u)} \mid u \in C(\Delta X)\right\}$ and $\mathcal{A}_{f}:=\{u \in C(\Delta X) \mid u$ is functional $\}$. Then $C(\Delta X)$ is the disjoint union of $\mathcal{A}_{d}$ and $\mathcal{A}_{f}$, that is, $C(\Delta X)=\mathcal{A}_{d} \sqcup \mathcal{A}_{f}$.

(b) We have $C\left(\Delta_{n} X\right)=\left(\mathcal{A}_{d} \cap C\left(\Delta_{n} X\right)\right) \sqcup\left(\mathcal{A}_{f} \cap C\left(\Delta_{n} X\right)\right)$.

Proof. (a) First we show that $\mathcal{A}_{d} \cap \mathcal{A}_{f}=\emptyset$. Let $\overline{d_{X}(u)} \in \mathcal{A}_{d}$ with $u \in C(\Delta X)$. Suppose the standard expression of $u$ is $\hat{u}\left(x^{(\ell)}\right)^{m}$ for some $\hat{u} \in C(\Delta X)$. Thus

$$
\overline{d_{X}(u)}=\overline{\hat{u} d_{X}\left(\left(x^{(\ell)}\right)^{m}\right)}=\overline{\hat{u}\left(x^{(\ell)}\right)^{m-1} x^{(\ell+1)}}=\overline{\hat{u}}\left(x^{(\ell)}\right)^{m-1} x^{(\ell+1)}
$$

and so $\overline{d_{X}(u)} \notin \mathcal{A}_{f}$. Next we show that $C(\Delta X)=\mathcal{A}_{d} \cup \mathcal{A}_{f}$. Let $u \in C(\Delta X) \backslash \mathcal{A}_{f}$. Suppose the minimum variable in $u$ is $x$ and $\ell$ is the largest differential degree of $x$. Then $u$ can be expressed 
as $u=\hat{u}\left(x^{(\ell-1)}\right)^{m} x^{(\ell)}$ with $\hat{u} \in C(\Delta X)$ and $m \geq 0$. Let $v=\hat{u}\left(x^{(\ell-1)}\right)^{m+1} \in C(\Delta X)$. By Lemma 5.6, we have that $u=\overline{d_{X}(v)} \in \mathcal{A}_{d}$. Hence $C(\Delta X)=\mathcal{A}_{d} \sqcup \mathcal{A}_{f}$.

(b) Since $C\left(\Delta_{n} X\right) \subseteq C(\Delta X)$, the result holds from Item (a).

Theorem 5.11. Let $A_{n}, A_{n, f}$ be as defined in Lemma 5.9 and let $I_{I D, n}$ be the differential RotaBaxter ideal of $\amalg\left(A_{n}\right)$ generated by $S_{n}$ in Eq. (28). Then as tensor product of modules

$$
\amalg\left(A_{n}\right) / I_{I D, n} \cong A_{n} \oplus\left(\bigoplus_{k \geq 0} A_{n} \otimes A_{n, f}^{\otimes k} \otimes A_{n}\right) .
$$

Proof. For any $s=\phi(u, v) \in S_{n}$, by Lemma 5.2, we have $\bar{s}=1 \otimes \overline{d\left(u_{1}\right)} \otimes w$, where $\overline{d\left(u_{1}\right)} \in$ $\mathcal{A}_{d} \cap C\left(\Delta_{n} X\right)$ and $w \in \mathcal{B}\left(\Delta_{n} X\right)$. Recall that

$$
\mathcal{B}\left(\Delta_{n} X\right)=\sqcup_{m \geq 1} C\left(\Delta_{n} X\right)^{\otimes m}=\left\{a_{1} \otimes \cdots \otimes a_{m} \mid a_{1}, \cdots, a_{m} \in C\left(\Delta_{n} X\right), m \geq 1\right\} .
$$

By Theorems 4.8 and 5.5, and Lemmas 5.9 and 5.10, we have

$$
\begin{aligned}
\operatorname{Irr}\left(\mathrm{S}_{\mathrm{n}}\right) & =\mathcal{B}\left(\Delta_{n} X\right) \backslash\left\{\left.q\right|_{\bar{s}}\left|q \in \mathcal{B}^{\star}\left(\Delta_{n} X\right), s \in S_{n}, q\right|_{s} \text { is normal }\right\} \\
& =\mathcal{B}\left(\Delta_{n} X\right) \backslash\left\{\left.q\right|_{1 \otimes \overline{d\left(u_{1}\right)} \otimes w} \mid q \in \mathcal{B}^{\star}\left(\Delta_{n} X\right), \overline{d\left(u_{1}\right)} \in \mathcal{A}_{d} \cap C\left(\Delta_{n} X\right), w \in \mathcal{B}\left(\Delta_{n} X\right)\right\} \\
& =\mathcal{B}\left(\Delta_{n} X\right) \backslash\left\{a_{1} \otimes \cdots \otimes a_{k} \in C\left(\Delta_{n} X\right)^{\otimes k} \mid a_{i} \in \mathcal{A}_{d} \cap C\left(\Delta_{n} X\right) \text { for some } 1<i<k, k \geq 1\right\} \\
& =\left\{a_{1} \otimes \cdots \otimes a_{k} \mid a_{1}, a_{k} \in C\left(\Delta_{n} X\right), a_{i} \in \mathcal{A}_{f} \cap C\left(\Delta_{n} X\right) \text { for } 1<i<k, k \geq 1\right\}
\end{aligned}
$$

is a k-basis of $\mathbf{k} \mathcal{B}\left(\Delta_{n} X\right) / I_{I D}$. Since $A_{n}=\mathbf{k} C\left(\Delta_{n} X\right)$ and $A_{n, f}=\mathbf{k} \mathcal{A}_{f} \cap C\left(\Delta_{n} X\right)$, the theorem follows.

Let

(38) $S:=\left\{P(d(u) P(v))-u P(v)+P(u v)+\lambda P(d(u) v) \mid u \in \amalg\left(\Delta_{n} X\right) \backslash P\left(\amalg\left(\Delta_{n} X\right)\right), v \in \amalg(\Delta X)\right\}$.

Lemma 5.12. Let $I_{I D, n}$ (resp. $\left.I_{I D}\right)$ be the differential Rota-Baxter ideal of $\amalg\left(\Delta_{n} X\right)($ resp. $\amalg(\Delta X))$ generated by $S_{n}$ (resp. $S$ ). Then as k-modules we have $I_{I D, 1} \subseteq I_{I D, 2} \subseteq \cdots, I_{I D}=\cup_{n \geq 1} I_{I D, n}$ and $I_{I D, n}=I_{I D} \cap \mathbf{k} \amalg\left(\Delta_{n} X\right)$.

Proof. Since $\mathbf{k} \amalg\left(\Delta_{n} X\right) \subseteq \mathbf{k} \amalg\left(\Delta_{n+1} X\right)$ for any $n \geq 1$, we have $I_{I D, 1} \subseteq I_{I D, 2} \subseteq \cdots$ and $I_{I D}=\cup_{n \geq 1} I_{I D, n}$ by Eq. (28). We next show $I_{I D, n}=I_{I D} \cap \amalg\left(\Delta_{n} X\right)$. Obviously, $I_{I D, n} \subseteq I_{I D} \cap \amalg\left(\Delta_{n} X\right)$. So we only need to verify $I_{I D} \cap \amalg\left(\Delta_{n} X\right) \subseteq I_{I D, n}$. By Theorem 5.11 we have

$$
\amalg\left(\Delta_{n} X\right) \cong\left(A_{n} \oplus\left(\bigoplus_{k \geq 0} A_{n} \otimes A_{n, f}^{\otimes k} \otimes A_{n}\right)\right) \oplus I_{I D, n} .
$$

Let

$$
J_{n}:=A_{n} \oplus\left(\bigoplus_{k \geq 0} A_{n} \otimes A_{n, f}^{\otimes k} \otimes A_{n}\right) .
$$

Then $\amalg\left(\Delta_{n} X\right)=J_{n} \oplus I_{I D, n}$ and $J_{1} \subseteq J_{2} \subseteq \cdots$. Let $n, k \geq 1$. Since $J_{n+k} \cap I_{I D, n+k}=0$ and $J_{n} \subseteq J_{n+k}$, we have $J_{n} \cap I_{I D, n+k}=0$. Since $I_{I D, n} \subseteq I_{I D, n+k}$, by modular law we have

$$
I_{I D, n+k} \cap \amalg\left(\Delta_{n} X\right)=I_{I D, n+k} \cap\left(J_{n} \oplus I_{I D, n}\right)=\left(I_{I D, n+k} \cap J_{n}\right) \oplus I_{I D, n}=I_{I D, n} .
$$

Let $u \in I_{I D} \cap \amalg\left(\Delta_{n} X\right)$. By $I_{I D}=\cup_{n \geq 1} I_{I D, n}$, we have $u \in I_{I D, N}$ for some $N \in \mathbb{Z}_{\geq 1}$. If $N \geq n$, by Eq. (39), $u \in I_{I D, N} \cap \amalg\left(\Delta_{n} X\right)=I_{I D, n}$. If $N<n$, then $u \in I_{I D, N} \subseteq I_{I D, n}$. Hence $I_{I D} \cap \amalg\left(\Delta_{n} X\right) \subseteq I_{I D, n}$ and so $I_{I D} \cap \amalg\left(\Delta_{n} X\right)=I_{I D, n}$.

Now we are ready to prove the main result of this paper. 
Theorem 5.13. (=Theorem 1.1) Let $X$ be a nonempty well-ordered set. Let $\amalg(\mathbf{k}\{X\})=\amalg(\Delta X)$ be the free commutative differential Rota-Baxter algebra on $X$. Let $I_{I D}$ be the ideal of $\amalg(\mathbf{k}\{X\})$ generated by $S$ defined in Eq. (38). Then the composition

$$
\amalg(A)_{f}:=A \oplus\left(\bigoplus_{k \geq 0} A \otimes A_{f}^{\otimes k} \otimes A\right) \hookrightarrow \amalg(A) \rightarrow \amalg(A) / I_{I D}
$$

of the inclusion and the quotient map is a linear isomorphism. In other words,

$$
\amalg(A)=\amalg(A)_{f} \oplus I_{I D} .
$$

Proof. First assume that $X$ is finite. Denote $A=\mathbf{k}[\Delta X]$ and $A_{n}=\mathbf{k}\left[\Delta_{n} X\right], n \geq 1$. By Theorem 5.11 and Lemma 5.12 we have the linear isomorphisms

$$
A_{n} \oplus\left(\bigoplus_{k \geq 0} A_{n} \otimes A_{n, f}^{\otimes k} \otimes A_{n}\right) \cong \amalg\left(\Delta_{n} X\right) / I_{I D, n}=\amalg\left(\Delta_{n} X\right) /\left(I_{I D} \cap \amalg\left(\Delta_{n} X\right)\right) \cong\left(\amalg\left(\Delta_{n} X\right)+I_{I D}\right) / I_{I D}
$$

that are compatible with the direct system $\underset{\longrightarrow}{\lim } A_{n}$. Since $A=\underset{\longrightarrow}{\lim } A_{n}$ as k-module, we have

$A \oplus\left(\bigoplus_{k \geq 0} A \otimes A_{f}^{\otimes k} \otimes A\right)=\underset{\lim }{\longrightarrow}\left(A_{n} \oplus\left(\bigoplus_{k \geq 0} A_{n} \otimes A_{n, f}^{\otimes k} \otimes A_{n}\right)\right) \cong \underset{\lim }{\longrightarrow}\left(\left(\amalg\left(\Delta_{n} X\right)+I_{I D}\right) / I_{I D}\right)=\amalg(A) / I_{I D}$.

Now let $X$ be a nonempty well-ordered set. Let $Y$ be a finite subset of $X$. Denote $A_{X, f}=$ $A_{f}, A_{Y, f}=\mathbf{k}\{Y\}_{f}$. Then by the definition of $A_{f}$ we have

$$
A_{Y} \cap A_{X, f}=A_{Y, f} \text { and } d_{X}\left(A_{Y}\right)=d_{Y}\left(A_{Y}\right) .
$$

Let $a \in A_{X}$. Then there is a finite $Y \subseteq X$ such that $a \in A_{Y}$. Thus by Proposition 5.8, we have $a \in A_{Y, f}+d_{X}\left(A_{Y}\right)$ which is contained in $A_{X, f}+d_{X}\left(A_{X}\right)$ by Eq. (40). Thus $A_{X}=A_{X, f}+d_{X}\left(A_{X}\right)$. On the other hand, let $0 \neq a \in d_{X}\left(A_{X}\right)$. Then $a=d_{X}(b)$ for $b \in A_{X}$. Then there is a finite $Y \subseteq X$ such that $b \in A_{Y}$ and hence $a \in d_{Y}\left(A_{Y}\right)$. Then by Proposition 5.8 and Eq. (40), we have $a \notin A_{Y, f}=A_{Y} \cap A_{X, f}$. Hence $a \notin A_{X, f}$. This proves $A_{X, f} \cap d_{X}\left(A_{X}\right)=0$. Hence $A_{X}=A_{X, f} \oplus d_{X}\left(A_{X}\right)$.

Now let $u \in \amalg\left(A_{X}\right)$. Then there is a finite subset $Y \subseteq X$ such that $a \in \amalg\left(A_{Y}\right)$. Then by the case of finite sets proved above, $u \in \amalg\left(A_{Y}\right)_{f}+I_{Y, I D}$. By definition, $\amalg\left(A_{Y}\right)_{f} \subseteq \amalg(A)_{f}$ and $I_{Y, I D} \subseteq I_{I D}$. Hence $u \in \amalg(A)_{f}+I_{I D}$. Further, if $0 \neq u \in I_{I D}$, then there is a finite $Y \subseteq X$ such that $u \in I_{Y, I D}$. Thus $u$ is not in $\amalg\left(A_{Y}\right)_{f}$ since $\amalg\left(A_{Y}\right)_{f} \cap I_{Y, I D}=0$. By the definition of $\amalg\left(A_{X}\right)_{f}$, we have $\amalg\left(A_{Y}\right) \cap \amalg\left(A_{X}\right)_{f}=\amalg\left(A_{Y}\right)_{f}$. Therefore $u$ is not in $\amalg\left(A_{X}\right)_{f}$. This proves $\amalg\left(A_{X}\right)=\amalg\left(A_{X}\right)_{f} \oplus I_{X, I D}$.

Acknowledgements: Xing Gao thanks support from NSFC grant 11201201. Li Guo acknowledges support from NSF grant DMS 1001855.

\section{REFERENCES}

[1] F. Baader T. and Nipkow, Term Rewriting and All That, Cambridge U. P., Cambridge, 1998. 8, 9, 11

[2] C. Bai, A unified algebraic approach to classical Yang-Baxter equation, J. Phys. A 40 (2007), 1107311082.2

[3] G. Baxter, An analytic problem whose solution follows from a simple algebraic identity, Pacific J. Math. 10 (1960), 731-742. 2

[4] G. M. Bergman, The diamond lemma for ring theory, Adv. Math. 29 (1978), 178-218. 3

[5] L. A. Bokut, Imbeddings into simple associative algebras, Algebra i Logika 15 (1976), 117-142. 3

[6] L. A. Bokut, Y. Chen, Y. Chen, Composition-Diamond lemma for tensor product of free algebras, J. Algebra 323 (2010), 2520-2537. 3 
[7] L. A. Bokut, Y. Chen and X. Deng, Gröbner-Shirshov bases for Rota-Baxter algebras, Siberian Math. J. 51 (2010), 978-988. 3

[8] L. A. Bokut, Y. Chen and Y. Li, Gröbner-Shirshov bases for categories, in "Operads and Universal Algebra", World Scientific Press, (2012) 1-23. 3

[9] L. A. Bokut, Y. Chen and J. Qiu, Greobner-Shirshov bases for associative algebras with multiple operators and free Rota-Baxter algebras, J. Pure Appl. Algebra 214 (2010) 89-110. 3, 6

[10] L.A. Bokut, V. Latyshev, I. Shestakov, E. Zelmanov (Eds.), Selected Works of A.I. Shirshov, Birkhäser, Basel, Boston, Berlin, 2009, vii, 241 pp. transl. M. Bremner, M. Kotchetov. 3

[11] B. Buchberger, An algorithm for finding a basis for the residue class ring of a zero-dimensional polynomial ideal [in German], Ph.D. thesis, University of Innsbruck, Austria, (1965). 3

[12] P. Cartier, On the structure of free Baxter algebras, Adv. Math. 9 (1972), 253-265. 2

[13] P. Cassidy, L.Guo, W. Keigher, W. Sit (Eds.), Differential Algebra and Related Topics (Proceedings for the International Workshop in Newark, NJ, 2000), World Sci. Publishing, 2002. 1

[14] P. M. Cohn, Algebra, Vol. 3, 2nd ed., J. Wiley \& Sons, Chichester, 1991.8

[15] K. Ebrahimi-Fard and L. Guo, Mixable shuffles, quasi-shuffles and Hopf algebras, J. Algebraic Combinatorics, 24 (2006), 83-101, arXiv:math.RA/0506418. 5

[16] K. Ebrahimi-Fard, L. Guo and D. Kreimer, Spitzer's identity and the algebraic Birkhoff decomposition in pQFT, J. Phys. A: Math. Gen. 37 (2004), 11037-11052. 2

[17] L. Guo, Operated semigroups, Motzkin paths and rooted trees, J. Algebraic Combin. 29 (2009), 35-62. 6,7

[18] L. Guo, WHAT IS a Rota-Baxter algebra, Notice of Amer. Math. Soc. 56 (2009) 1436-1437. 2

[19] L. Guo, Introduction to Rota-Baxter Algebra, Higher Education Press and International Press, 2012. 2, $5,6,7$

[20] L. Guo and W. Keigher, Baxter algebras and shuffle products, Adv. Math., 150 (2000), 117-149, arXiv:math/0407155.2, 5, 6

[21] L. Guo and W. Keigher, On free Baxter algebras: completions and the internal construction, Adv. Math. 151 (2000), 101-127, arXiv:math/0407156. 5, 6

[22] L. Guo and W. Keigher, On differential Rota-Baxter algebras, J. Pure Appl. Algebra, 212 (2008), 522540. 2, 3, 5, 6

[23] L. Guo, G. Regensburger and M. Rosenkranz, On integro-differential algebras, arXiv:1212.0266. 2, 3, 4, 24

[24] L. Guo, W. Sit and R. Zhang, Differemtail type operators and Gröbner-Shirshov bases, J. Symolic Comput. (2012). 6

[25] L. Guo and B. Zhang, Renormalization of multiple zeta values, J. Algebra, 319 (2008), 3770-3809. 2,5

[26] H. Hironaka, Resolution of singulatities of an algebraic variety over a field if characteristic zero, I, Ann. Math. 79 (1964), 109-203. 3

[27] M. Hoffman, Quasi-shuffle products, J. Algebraic Combin., 11 (2000), 49-68. 2, 5

[28] E. R. Kolchin, Differential Algebras and Algebraic Groups, Academic Press, New York, 1973. 1

[29] J. Qiu, Gröbner-Shirshov bases for commutative algebras with multiple operators and free commutative Rota-Baxter algebras, Asian-European Jour. Math., to appear. 6

[30] J.F. Ritt, Differential Equations from the Algebraic Standpoint, Amer. Math. Sco. Colloq. Pub. 14, Amer. Math. Soc., New York (1934). 1

[31] J.F. Ritt, Differential Algebra, Amer. Math. Sco. Colloq. Pub. 33, Amer. Math. Soc., New York (1950). 1

[32] M. Rosenkranz and G. Regensburger, Solving and factoring boundary problems for linear ordinary differential equations in differential algebra, J. Symbolic Comput. 43 (2008), 515-544. 2

[33] G.-C. Rota, Baxter algebras and combinatorial identities I, II, Bull. Amer. Math. Soc. 75 (1969), 325$329,330-334.2$

[34] G.-C. Rota, Baxter operators, an introduction, In: "Gian-Carlo Rota on Combinatorics, Introductory papers and commentaries", Joseph P. S. Kung, Editor, Birkhäuser, Boston, 1995. 2

[35] M. A. Semenov-Tian-Shansky, What is a classical $r$-matrix?, Funct. Ana. Appl., 17 (1983) 259-272. 2

[36] A. I. Shirshov, Some algorithmic problem for Å-algebras, Sibirsk. Mat. Z., 3(1962), 132-137. 3

[37] M. Singer and M. van der Put, Galois Theory of Linear Differential Equations, Springer, 2003. 1 
Department of Mathematics, Lanzhou University, Lanzhou, Gansu 730000, China

E-mail address: gaoxing@lzu.edu.cn

Department of Mathematics and Computer Science, Rutgers University, Newark, NJ 07102, USA

E-mail address: liguo@rutgers.edu

Department of Mathematics, Lanzhou University, Lanzhou, Gansu 730000, China

E-mail address: zheng2712801@163.com 